\title{
X-ray Properties of Black-Hole Binaries
}

\author{
Ronald A. Remillard \\ Kavli Institute for Astrophysics and Space Research, MIT, Cambridge, Massachusetts 02139 \\ email: rr@space.mit.edu
}

Jeffrey E. McClintock

Harvard-Smithsonian Center for Astrophysics, Cambridge, Massachusetts 02138 email:jem@cfa.harvard.edu

KEYWORDS: accretion physics, black holes, general relativity, X-ray Sources

\begin{abstract}
We review the properties and behavior of $20 \mathrm{X}$-ray binaries that contain a dynamically-confirmed black hole, 17 of which are transient systems. During the past decade, many of these transient sources were observed daily throughout the course of their typically year-long outburst cycles using the large-area timing detector aboard the Rossi X-ray Timing Explorer. The evolution of these transient sources is complex. Nevertheless, there are behavior patterns common to all of them as we show in a comprehensive comparison of six selected systems. Central to this comparison are three X-ray states of accretion, which are reviewed and defined quantitatively. We discuss phenomena that arise in strong gravitational fields, including relativistically-broadened Fe lines, high-frequency quasi-periodic oscillations (100-450 Hz), and relativistic radio and X-ray jets. Such phenomena show us how a black hole interacts with its environment, thereby complementing the picture of black holes that gravitational wave detectors will provide. We sketch a scenario for the potential impact of timing/spectral studies of accreting black holes on physics and discuss a current frontier topic, namely, the measurement of black hole spin.
\end{abstract}

\section{CONTENTS}

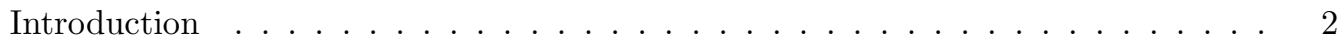

A Census of Black-Hole Binaries and Black-Hole Candidates . . . . . . . . . . . 3

X-ray Observations of Black-Hole Binaries . . . . . . . . . . . . . . . . . 6 X-ray Light Curves of Black-Hole Binaries in Outburst . . . . . . . . . . . . . . . . . . 6 X-ray Timing . . . . . . . . . . . . . . . . . . . . . . . 7 X-ray Spectra . . . . . . . . . . . . . . . . . . . . 7

Emission States of Black-Hole Binaries . . . . . . . . . . . . . . . . . . 8 Historical Notes on X-ray States . . . . . . . . . . . . . . . . . . . . 8 A Quantitative Three-State Description for Active Accretion . . . . . . . . . . . . . . 9 The Unified Model for Radio Jets . . . . . . . . . . . . . . . . . . . . . . . . . . 11 Quiescent State . . . . . . . . . . . . . . . . . . 13

X-ray Overview of State Evolution and Energetics . . . . . . . . . . . . . . 13 Overview Plots for Six Individual Sources . . . . . . . . . . . . . . . . . . . . . . . . . 13 
Conclusions from Overviews of X-ray States . . . . . . . . . . . . . . . . . . . 21

X-ray Quasi-Periodic Oscillations . . . . . . . . . . . . . . . . . . . 22

Low-Frequency Quasi-Periodic Oscillations . . . . . . . . . . . . . . . . . . 22

High-Frequency Quasi-Periodic Oscillations . . . . . . . . . . . . . . . . . . 24

Physical Models for X-ray States . . . . . . . . . . . . . . . . . . . 26

Thermal State. . . . . . . . . . . . . . . . . . 26

Hard State. . . . . . . . . . . . . . . . . . . . 27

Steep Power Law State . . . . . . . . . . . . . . . . . . . . . . . . . . . . 28

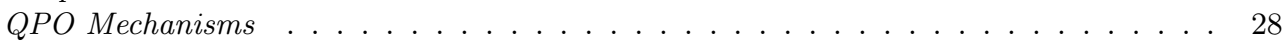

Accreting Black Holes as Probes of Strong Gravity . . . . . . . . . . . . . . . . . . 29

Black Holes Binaries: The Journey from Astrophysics to Physics . . . . . . . . . . . . 29

Measuring Black Hole Spin: A Current Frontier. . . . . . . . . . . . . . . . . 30

Critique of Methods for Measuring Spin . . . . . . . . . . . . . . . . . . . . . 34

Black-Hole Binaries Yesterday, Today and Tomorrow . . . . . . . . . . . . . . . . . 34

Acknowledgements; Acronyms; Section Summaries . . . . . . . . . . . . . . . 35

\section{Introduction}

Oppenheimer \& Snyder (1939) made the first rigorous calculation describing the formation of a black hole (BH). The first strong evidence for such an object came from X-ray and optical observations of the X-ray binary Cygnus X-1 (Bolton 1972; Webster \& Murdin 1972). Today, a total of 20 similar X-ray binary systems are known that contain a compact object believed to be too massive to be a neutron star or a degenerate star of any kind (i.e., $M>3 M_{\odot}$ ). These systems, which we refer to as black-hole binaries (BHBs), are the focus of this review.

These 20 dynamical $\mathrm{BHs}$ are the most visible representatives of an estimated $10^{8}-10^{9}$ stellar-mass BHs that are believed to exist in the Galaxy (e.g., Brown \& Bethe 1994; Timmes, Woosley, \& Weaver 1996). Stellar-mass BHs are important to astronomy in numerous ways. For example, they are one endpoint of stellar evolution for massive stars, and the collapse of their progenitor stars enriches the universe with heavy elements (Woosley et al. 2002). Also, the measured mass distribution for even the small sample featured here is used to constrain models of BH formation and binary evolution (e.g., Fryer \& Kalogera 2001; Podsiadlowski et al. 2003). Lastly, some BHBs appear to be linked to the hypernovae believed to power gamma-ray bursts (Israelian et al. 1999; Brown et al. 2000; Orosz et al. 2001).

The BHBs featured here are mass-exchange binaries that contain an accreting $\mathrm{BH}$ primary and a nondegenerate secondary star. For background on X-ray binaries, see Psaltis (2006). For comprehensive reviews on BHBs, see McClintock \& Remillard (2006), Tanaka and Shibazaki (1996) and Tanaka \& Lewin (1995). X-ray observations of BHBs allow us to gain a better understanding of $\mathrm{BH}$ properties and accretion physics. In this review, we emphasize those results that challenge us to apply the predictions of general relativity (GR) in strong gravity (§8). Throughout, we make extensive use of the extraordinary data base amassed since January 1996 by NASA's Rossi X-ray Timing Explorer (RXTE; Swank 1998).

In an astrophysical environment, a $\mathrm{BH}$ is completely specified in GR by two 
numbers, its mass $M$ and its specific angular momentum or spin $a=J / c M$, where $J$ is the $\mathrm{BH}$ angular momentum and $c$ is the speed of light. The spin value is conveniently expressed in terms of a dimensionless spin parameter, $a_{*}=a / R_{\mathrm{g}}$, where the gravitational radius is $R_{\mathrm{g}} \equiv G M / c^{2}$. The mass simply supplies a scale, whereas the spin changes the geometry. The value of $a_{*}$ lies between 0 for a Schwarzschild hole and 1 for a maximally-rotating Kerr hole. A defining property of a $\mathrm{BH}$ is its event horizon, the immaterial surface that bounds the interior region of space-time that cannot communicate with the external universe. The event horizon, the existence of an innermost stable circular orbit (ISCO), and other properties of BHs are discussed in many texts (e.g., Shapiro \& Teukolsky 1983; Kato et al. 1998). The radius of the event horizon of a Schwarzschild BH $\left(a_{*}=0\right)$ is $R_{\mathrm{S}}=2 R_{\mathrm{g}}=30 \mathrm{~km}\left(M / 10 M_{\odot}\right)$, the ISCO lies at $R_{\mathrm{ISCO}}=6 R_{\mathrm{g}}$, and the corresponding maximum orbital frequency is $\nu_{\mathrm{ISCO}}=220 \mathrm{~Hz}\left(M / 10 M_{\odot}\right)^{-1}$. For an extreme Kerr BH $\left(a_{*}=1\right)$, the radii of both the event horizon and the ISCO (prograde orbits) are identical, $R_{\mathrm{K}}=R_{\mathrm{ISCO}}=R_{\mathrm{g}}$, and the maximum orbital frequency is $\nu_{\mathrm{ISCO}}=1615 \mathrm{~Hz}\left(M / 10 M_{\odot}\right)^{-1}$.

\section{A Census of Black-Hole Binaries and Black-Hole Candidates}

Following the discovery of Cygnus $\mathrm{X}-1$, the second BHB to be identified was LMC X-3 (Cowley et al. 1983). Both sources are persistently bright in X-rays, and their secondaries are massive O/B-type stars (White et al. 1995). The third identified BHB, A 0620-00, is markedly different (McClintock \& Remillard 1986). A 0620-00 was discovered as an X-ray nova in 1975 when it suddenly brightened to an intensity of $50 \mathrm{Crab}^{1}$ to become the brightest nonsolar X-ray source ever observed (Elvis et al. 1975). Then, over the course of a year, the X-ray nova decayed back into quiescence to become a feeble $(1 \mu \mathrm{Crab})$ source (McClintock et al. 1995). Similarly, the optical counterpart faded from outburst maximum by $\Delta V \approx 7.1$ mags to $V \approx 18.3$ in quiescence, thereby revealing the optical spectrum of a K-dwarf secondary.

As of this writing, there are a total of 20 confirmed BHBs and, remarkably, 17 of them are X-ray novae like A $0620-00$. They are ordered in the top half of Table 1 by right ascension (column 1). Column 2 gives the common name of the source (e.g., LMC X-3) or the prefix to the coordinate name that identifies the discovery mission (e.g., XTE J, where a "J" indicates that the coordinate epoch is J2000). For X-ray novae, the third column gives the year of discovery and the number of outbursts that have been observed. The spectral type of the secondary star is given in column 4. Extensive optical observations of this star yield the key dynamical data summarized respectively in the last three columns: the orbital period, the mass function, and the $\mathrm{BH}$ mass. Additional data on BHBs are given in Tables 4.1 \& 4.2 of McClintock \& Remillard (2006).

An observational quantity of special interest is the mass function, $f(M) \equiv$ $P_{\text {orb }} K_{2}^{3} / 2 \pi G=M_{1} \sin ^{3} i /(1+q)^{2}$ (see Table 1, column 6 ). The observables on the left side of this equation are the orbital period $P_{\text {orb }}$ and the half-amplitude of the velocity curve of the secondary $K_{2}$. On the right, the quantity of greatest interest is $M_{1}$, the mass of the $\mathrm{BH}$ primary (given in column 7 ); the other parameters are the orbital inclination angle $i$ and the mass ratio $q \equiv M_{2} / M_{1}$, where $M_{2}$

\footnotetext{
${ }^{1} 1 \mathrm{Crab}=2.43 \times 10^{-9} \mathrm{erg} \mathrm{\textrm {cm } ^ { - 2 }} \mathrm{s}^{-1} \mathrm{keV}^{-1}=1.00 \mathrm{mJy} \quad$ (averaged over 2-11 keV) for a Crab-like spectrum with photon index $\Gamma=2.08$; Koyama et al. (1984).
} 
is the mass of the secondary. The value of $f(M)$ can be determined by simply measuring the radial velocity curve of the secondary star, and it corresponds to the absolute minimum allowable mass of the compact object.

An inspection of Table 1 shows that 15 of the 20 X-ray sources have values of $f(M)$ that require a compact object with a mass $\gtrsim 3 M_{\odot}$. This is a widely agreed limit for the maximum stable mass of a neutron star in GR (e.g., Kalogera \& Baym 1996). For the remaining five systems, some additional data are required to make the case for a BH (Charles \& Coe 2006; McClintock \& Remillard 2006). Historically, the best available evidence for the existence of BHs is dynamical, and the evidence for these 20 systems is generally very strong, with cautions for only two cases: LMC X-1 and XTE J1859+226 (see McClintock \& Remillard 2006). Thus, assuming that GR is valid in the strong-field limit, we choose to refer to these compact primaries as BHs, rather than as $\mathrm{BH}$ candidates.

\section{Black Hole Binaries in the Milky Way}

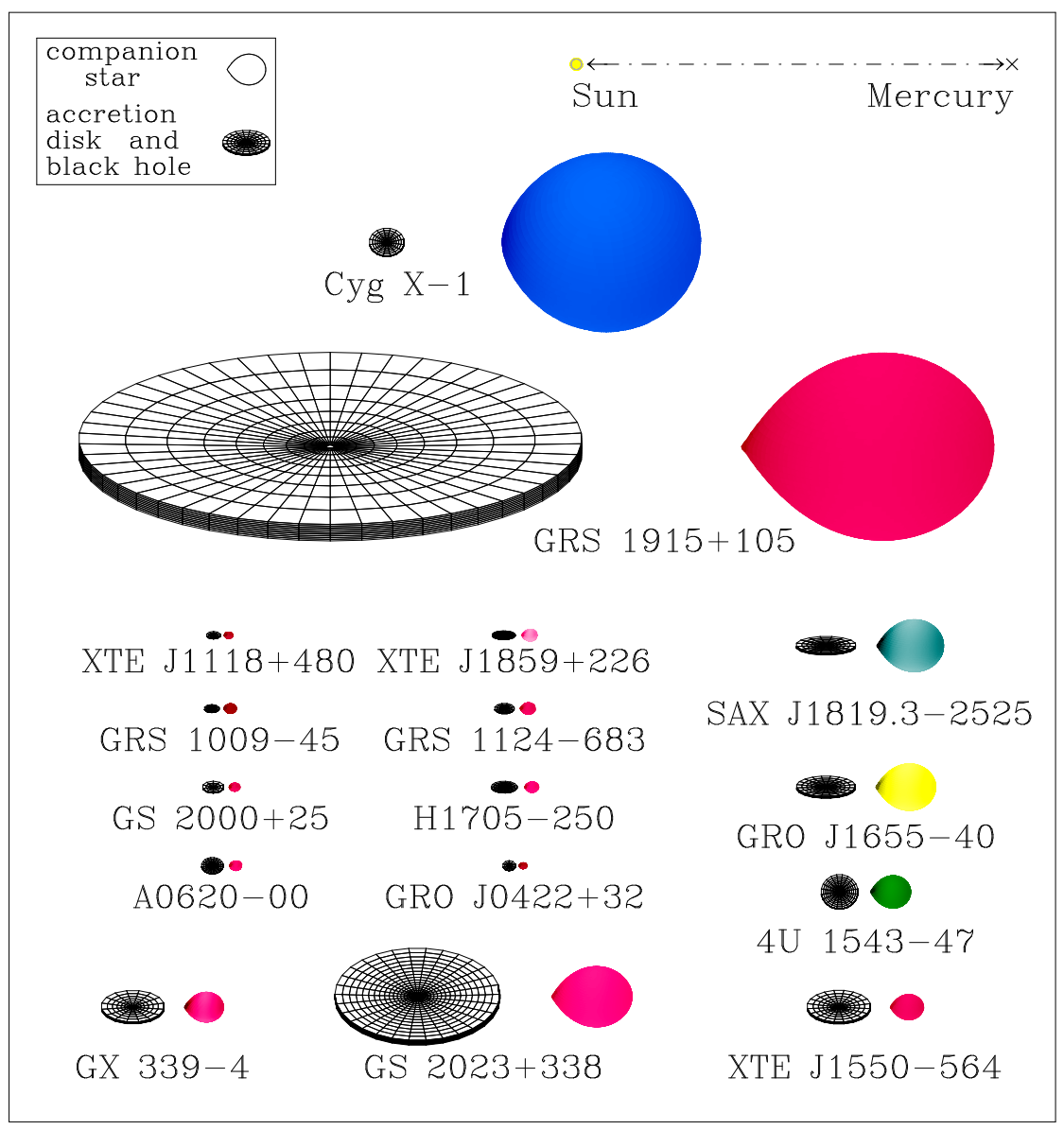

Figure 1: Scale drawings of 16 black-hole binaries in the Milky Way (courtesy of J. Orosz). The Sun-Mercury distance $(0.4 \mathrm{AU})$ is shown at the top. The estimated binary inclination is indicated by the tilt of the accretion disk. The color of the companion star roughly indicates its surface temperature.

Figure 1 is a schematic sketch of 16 Milky Way BHBs with reasonably accurate dynamical data. Their diversity is evident: there are long-period systems 
Table 1: Twenty confirmed black holes and twenty black hole candidates ${ }^{a}$

\begin{tabular}{|c|c|c|c|c|c|c|}
\hline $\begin{array}{l}\text { Coordinate } \\
\text { Name }\end{array}$ & $\begin{array}{l}\text { Common }^{b} \\
\text { Name/Prefix }\end{array}$ & Year $^{c}$ & Spec. & $\begin{array}{l}\mathrm{P}_{\text {orb }} \\
(\mathrm{hr})\end{array}$ & $\begin{array}{l}\mathrm{f}(\mathrm{M}) \\
\left(\mathrm{M}_{\odot}\right)\end{array}$ & $\begin{array}{c}\mathrm{M}_{1} \\
\left(\mathrm{M}_{\odot}\right)\end{array}$ \\
\hline $0422+32$ & $($ GRO J) & $1992 / 1$ & $\mathrm{M} 2 \mathrm{~V}$ & 5.1 & $1.19 \pm 0.02$ & $3.7-5.0$ \\
\hline $0538-641$ & LMC X-3 & - & B3V & 40.9 & $2.3 \pm 0.3$ & $5.9-9.2$ \\
\hline $0540-697$ & LMC X-1 & - & O7III & $93.8^{d}$ & $0.13 \pm 0.05^{d}$ & $4.0-10.0:^{e}$ \\
\hline 0620-003 & (A) & $1975 / 1^{f}$ & $\mathrm{~K} 4 \mathrm{~V}$ & 7.8 & $2.72 \pm 0.06$ & $8.7-12.9$ \\
\hline $1009-45$ & (GRS) & $1993 / 1$ & $\mathrm{~K} 7 / \mathrm{M} 0 \mathrm{~V}$ & 6.8 & $3.17 \pm 0.12$ & $3.6-4.7:^{e}$ \\
\hline $1118+480$ & (XTE J) & $2000 / 2$ & $\mathrm{~K} 5 / \mathrm{M} 0 \mathrm{~V}$ & 4.1 & $6.1 \pm 0.3$ & $6.5-7.2$ \\
\hline $1124-684$ & Nova Mus 91 & $1991 / 1$ & $\mathrm{~K} 3 / \mathrm{K} 5 \mathrm{~V}$ & 10.4 & $3.01 \pm 0.15$ & $6.5-8.2$ \\
\hline $1354-64^{g}$ & $(\mathrm{GS})$ & $1987 / 2$ & GIV & $61.1^{g}$ & $5.75 \pm 0.30$ & - \\
\hline $1543-475$ & $(4 \mathrm{U})$ & $1971 / 4$ & $\mathrm{~A} 2 \mathrm{~V}$ & 26.8 & $0.25 \pm 0.01$ & $8.4-10.4$ \\
\hline $1550-564$ & (XTE J) & $1998 / 5$ & G8/K8IV & 37.0 & $6.86 \pm 0.71$ & $8.4-10.8$ \\
\hline $1650-500^{h}$ & (XTE J) & $2001 / 1$ & $\mathrm{~K} 4 \mathrm{~V}$ & 7.7 & $2.73 \pm 0.56$ & - \\
\hline $1655-40$ & (GRO J) & $1994 / 3$ & F3/F5IV & 62.9 & $2.73 \pm 0.09$ & $6.0-6.6$ \\
\hline $1659-487$ & GX 339-4 & $1972 / 10^{i}$ & - & $42.1^{j, k}$ & $5.8 \pm 0.5$ & - \\
\hline $1705-250$ & Nova Oph 77 & $1977 / 1$ & $\mathrm{~K} 3 / 7 \mathrm{~V}$ & 12.5 & $4.86 \pm 0.13$ & $5.6-8.3$ \\
\hline $1819.3-2525$ & V4641 Sgr & $1999 / 4$ & B9III & 67.6 & $3.13 \pm 0.13$ & $6.8-7.4$ \\
\hline $1859+226$ & $(\mathrm{XTE} \mathrm{J})$ & $1999 / 1$ & - & $9.2:^{e}$ & $7.4 \pm 1.1:^{e}$ & $7.6-12.0:^{e}$ \\
\hline $1915+105$ & (GRS) & $1992 / \mathrm{Q}^{l}$ & K/MIII & 804.0 & $9.5 \pm 3.0$ & $10.0-18.0$ \\
\hline $1956+350$ & Cyg X-1 & - & O9.7Iab & 134.4 & $0.244 \pm 0.005$ & $6.8-13.3$ \\
\hline $2000+251$ & (GS) & 1988/1 & $\mathrm{K} 3 / \mathrm{K} 7 \mathrm{~V}$ & 8.3 & $5.01 \pm 0.12$ & $7.1-7.8$ \\
\hline $2023+338$ & V404 Cyg & $1989 / 1^{f}$ & K0III & 155.3 & $6.08 \pm 0.06$ & $10.1-13.4$ \\
\hline $1524-617$ & $(\mathrm{~A})$ & $1974 / 2$ & - & - & - & - \\
\hline $1630-472$ & $(4 \mathrm{U})$ & $1971 / 15$ & - & - & - & - \\
\hline 1711.6-3808 & $(\mathrm{SAX} J)$ & $2001 / 1$ & - & - & - & - \\
\hline 1716-249 & (GRS) & $1993 / 1$ & - & 14.9 & - & - \\
\hline $1720-318$ & (XTE J) & $2002 / 1$ & - & - & - & - \\
\hline $1730-312$ & $(\mathrm{KS})$ & $1994 / 1$ & - & - & - & - \\
\hline $1737-31$ & (GRS) & $1997 / 1$ & - & - & - & - \\
\hline 1739-278 & (GRS) & $1996 / 1$ & - & - & - & - \\
\hline $1740.7-2942$ & $(1 \mathrm{E})$ & - & - & - & - & - \\
\hline $1743-322$ & $(\mathrm{H})$ & $1977 / 4$ & - & - & - & - \\
\hline $1742-289$ & (A) & $1975 / 1$ & - & - & - & - \\
\hline $1746-331$ & $(\mathrm{SLX})$ & $1990 / 2$ & - & - & - & - \\
\hline $1748-288$ & (XTE J) & $1998 / 1$ & - & - & - & - \\
\hline $1755-324$ & (XTE J) & $1997 / 1$ & - & - & - & - \\
\hline $1755-338$ & $(4 \mathrm{U})$ & $1971 / \mathrm{Q}^{l}$ & - & 4.5 & - & - \\
\hline $1758-258$ & (GRS) & $1990 / \mathrm{Q}^{l}$ & - & - & - & - \\
\hline $1846-031$ & $(\mathrm{EXO})$ & $1985 / 1$ & - & - & - & - \\
\hline $1908+094$ & (XTE J) & $2002 / 1$ & - & - & - & - \\
\hline $1957+115$ & $(4 \mathrm{U})$ & - & - & 9.3 & - & - \\
\hline $2012+381$ & $(\mathrm{XTE} J)$ & $1998 / 1$ & - & - & - & - \\
\hline
\end{tabular}

${ }^{a}$ See McClintock \& Remillard (2006; and references therein) for columns 3-5, Orosz (2003) for columns 6-7, plus additional references given below.

${ }^{b} \mathrm{~A}$ prefix to a coordinate name is enclosed in parentheses. The presence/absence of a

"J"indicates that the epoch of the coordinates is J2000/B1950.

${ }^{c}$ Year of initial X-ray outburst/total number of X-ray outbursts.

${ }^{d}$ Period and $\mathrm{f}(\mathrm{M})$ corrections by AM Levine and D Lin, private communication.

${ }^{e}$ Colon denotes uncertain value or range.

${ }^{f}$ Additional outbursts in optical archives: A 0620 (1917) and V404 Cyg $(1938,1956)$.

${ }^{g}$ Casares et al. 2004; possible alias period of $61.5 \mathrm{hr}$.

${ }^{h}$ Orosz et al. 2004.

${ }^{i}$ Estimated by Kong et al. 2002.

${ }^{j}$ Hynes et al. 2003.

${ }^{k}$ Period confirmed by A.M. Levine and D. Lin, private communication.

$l$ "Q" denotes quasi-persistent intervals (e.g., decades), rather than typical outburst. 
containing hot and cool supergiants (Cyg X-1 and GRS 1915+105) and many compact systems containing K-dwarf secondaries. Considering all 20 BHBs (Table 1), only 3 are persistently bright X-ray sources (Cyg X-1, LMC X-1 and LMC $\mathrm{X}-3)$. The 17 transient sources include 2 that are unusual. GRS $1915+105$ has remained bright for more than a decade since its first known eruption in August 1992. GX 339-4 undergoes frequent outbursts followed by very faint states, but it has never been observed to fully reach quiescence (Hynes et al. 2003).

The second half of Table 1 lists 20 X-ray binaries that lack radial velocity data. In fact, most of them even lack an optical counterpart, and only three have known orbital periods. Nevertheless, they are considered black-hole candidates (BHCs) because they closely resemble BHBs in their X-ray spectral and temporal behavior (McClintock \& Remillard 2006; Tanaka \& Lewin 1995). Some X-ray and radio characteristics of the BHCs are given in Table 4.3 in McClintock \& Remillard (2006); also given there is a subjective grade (A, B or C) indicating the likelihood that a particular system does contain a $\mathrm{BH}$ primary. The seven A-grade BHCs are: A 1524-617, 4U 1630-47, GRS 1739-278, 1E 1740.7-2942, H 1743-322, XTE J1748-288, and GRS 1758-258.

\section{X-ray Observations of Black-Hole Binaries}

We first discuss X-ray light curves obtained by wide-angle X-ray cameras that are used to discover X-ray novae and to monitor hundreds of sources on a daily basis. We then discuss timing and spectral analyses of data obtained in pointed observations that reveal, in detail, the properties of accreting BHs. Throughout this work we feature RXTE results derived from the huge and growing archive of data amassed since 1996 by the All-Sky Monitor (ASM) and the large-area Proportional Counter Array (PCA) detector (Swank 1998).

\subsection{X-ray Light Curves of Black-Hole Binaries in Outburst}

Nearly all BHBs are X-ray novae (see $\S 2$ and Table 1) that are discovered when they first go into outburst. Their discovery and subsequent daily monitoring are largely dependent on wide-field X-ray cameras on orbiting satellites. The light curves of all 20 BHBs and many BHCs (Table 1) can be found either in McClintock \& Remillard (2006) or in a review paper on pre-RXTE X-ray novae by Chen et al. (1997). These researchers discuss the striking morphological diversity among these light curves, which show broad distributions in their timescales for rise and decay.

For X-ray outbursts that last between $\sim 20$-days and many months, the generally accepted cause of the outburst cycle is an instability that arises in the accretion disk. When the accretion rate from the donor star is not sufficient to support continuous viscous flow to the compact object, matter fills the outer disk until a critical surface density is reached and an outburst is triggered. This model was developed initially for dwarf novae (e.g., Smak 1971; Lasota 2001) and extended to X-ray novae (e.g., Dubus et al. 2001).

This model predicts recurrent outbursts; indeed, half of the BHBs are now known to recur on timescales of 1 to 60 years (Table 1). Outbursts on much shorter or longer timescales do occur, but these are not understood in terms of the disk instability model. Sources such as GRS 1915+105 and 4U 1755-338 exhibit "on" and "off" states that can persist for $\gtrsim 10$ years. The behavior of 
the companion star may play a role in causing these long-term changes in the accretion rate.

After a decade of continuous operation, the ASM continues to scan most of the celestial sphere several times per day (Levine et al. 1996). It has discovered 8 BHB/BHC X-ray novae and an additional 15 recurrent outbursts. Detailed X-ray light curves have been archived for each of these sources, and complete outbursts for six such systems are shown in $\S 5$.

\subsection{X-ray Timing}

Our most important resource for examining the near-vicinity of a $\mathrm{BH}$ is the rapid variations in X-ray intensity that are so often observed (van der Klis 2005; McClintock \& Remillard 2006). The analysis tool commonly used for probing fast variability is the power-density spectrum (PDS; e.g., Leahy et al. 1983). Related techniques for computing coherence and phase lag functions are reviewed by Vaughan \& Nowak (1997). PDSs are interpreted with the presumption that the source variations are a locally stationary process. More generalized considerations of time series analyses are given by Scargle (1981), while recent topics in nonlinear processes are well described by Gliozzi, Papadakis, \& Räth (2006).

The PDS is used extensively in this work. The continuum power in the PDS is of interest for both its shape and its integrated amplitude (e.g., 0.1-10 Hz), which is usually expressed in units of rms fluctuations scaled to the mean count rate. PDSs of BHBs also exhibit transient, discrete features known as quasi-periodic oscillations (QPOs) that may range in frequency from 0.01 to $450 \mathrm{~Hz}$. QPOs are generally modeled with Lorentzian profiles, and they are distinguished from broad power peaks using a coherence parameter, $Q=\nu / F W H M \gtrsim 2$ (Nowak 2000; van der Klis 2005). PDSs are frequently computed for a number of energy intervals. This is an important step in linking oscillations to an individual component in the X-ray spectrum.

\subsection{X-ray Spectra}

It has been known for decades that the energy spectra of BHBs often exhibit a composite shape consisting of both a thermal and a nonthermal component. Furthermore, BHBs display transitions in which one or the other of these components may dominate the X-ray luminosity (see Tanaka \& Lewin 1995; McClintock \& Remillard 2006). The thermal component is well modeled by a multitemperature blackbody, which originates in the inner accretion disk and often shows a characteristic temperature near $1 \mathrm{keV}$ (see $\S 7$ ). The nonthermal component is usually modeled as a power law (PL). It is characterized by a photon index $\Gamma$, where the photon spectrum is $N(E) \propto E^{-\Gamma}$. The PL generally extends to much higher photon energies $(E)$ than does the thermal component, and sometimes the PL suffers a break or an exponential cutoff at high energy.

X-ray spectra of BHBs may also exhibit an $\mathrm{Fe} \mathrm{K} \alpha$ emission line that is often relativistically broadened (§8.2.3). In some BHBs, particularly those with inclinations that allow us to view the disk largely face-on, the spectral model requires the addition of a disk reflection component (e.g., Done \& Nayakshin 2001). In this case, the X-ray PL is reflected by the accretion disk and produces a spectral bump at roughly 10 to $30 \mathrm{keV}$. Finally, high-resolution grating spectra of BHBs sometimes reveal hot gas that is local to the binary system (e.g., Lee et al 2002). 
Such features may eventually help us to interpret X-ray states, but at present there are too few results to support any firm conclusions.

\section{Emission States of Black-Hole Binaries}

\subsection{Historical Notes on X-ray States}

The concept of X-ray states was born when Tananbaum et al. (1972) observed a global spectral change in Cyg X-1 in which the soft X-ray flux (2-6 keV) decreased by a factor of 4 , the hard flux $(10-20 \mathrm{keV})$ increased by a factor of 2 , and the radio counterpart turned on. Thereafter, a similar X-ray transition was seen in A 062000 (Coe et al. 1976) and in many other sources as well. The soft state, which was commonly described as $\sim 1 \mathrm{keV}$ thermal emission, was usually observed when the source was bright, thereby prompting the name "high/soft state". The hard state, with a typical photon index $\Gamma \sim 1.7$, was generally seen when the source was faint, hence the name "low/hard state". In this state, the disk was either not observed above $2 \mathrm{keV}$, or it appeared much cooler and withdrawn from the BH. An additional X-ray state of BHBs was identified in the Ginga era (Miyamoto \& Kitamoto 1991; Miyamoto et al. 1993). It was characterized by the appearance of several-Hz X-ray QPOs, a relatively high luminosity (e.g., $>0.1 L_{\mathrm{Edd}}$ ), and a spectrum comprised of both a thermal component and a PL component that was steeper $(\Gamma \sim 2.5)$ than the hard PL. This state was named the "very high" state.

Rapid observational developments in the RXTE era challenged the prevailing views of X-ray states in BHBs. First, it was shown that the soft state of Cyg X-1 is not consistent with a thermal interpretation (Zhang et al. 1997b); instead, the spectrum is dominated by a steep PL component $(\Gamma \sim 2.5)$. Thus, Cyg $\mathrm{X}-1$ is not a useful prototype for the high/soft state that it helped to define. Secondly, the spectra of $\mathrm{BH}$ transients near maximum luminosity were often found to exhibit a steep PL spectrum, rather than a thermal spectrum (McClintock \& Remillard 2006). Thirdly, a number of different QPO types were commonly observed over a wide range of luminosities (e.g., Morgan et al. 1997; Sobczak et al. 2000a; Homan et al. 2001). These findings attracted great interest in the nature of the very high state.

During this period, Gamma-ray observations $(\sim 40-500 \mathrm{keV})$ of seven BHBs brought clarity to the distinction between the soft and hard types of X-ray PL components (Grove et al. 1998; Tomsick et al. 1999). Sources in the low/hard state $(\Gamma \sim 1.7)$ were found to suffer an exponential cutoff near $100 \mathrm{keV}$, whereas sources with soft X-ray spectra $(\Gamma \sim 2.5)$ maintained a steep, strong and unbroken PL component out to the sensitivity limit of the gamma-ray detectors $(\sim 1 \mathrm{MeV})$.

More recently, radio observations have cemented the association of the low/hard state with the presence of a compact and quasi-steady radio jet (see Fender 2006; McClintock \& Remillard 2006). In brief, the evidence for the association includes the following: $(a)$ the presence of compact jets in VLBI images of two BH sources (Dhawan, Mirabel, \& Rodriguez 2000; Stirling et al. 2001); (b) correlated X-ray and radio intensities and/or the presence of flat or inverted radio spectra (e.g., Gallo et al. 2003), which allow the jet's presence to be inferred even in the absence of VLBI images; $(c)$ a $2 \%$ linear radio polarization at nearly constant position angle observed for GX 339-4 (Corbel et al. 2000); and $(d)$ the frequently-observed

quenching of the persistent radio emission that occurs when a BHB switches from the low/hard state to the high/soft state (e.g., Fender et al. 1999). 


\subsection{A Quantitative Three-State Description for Active Accretion}

In McClintock \& Remillard (2006), a new framework was used to define X-ray states that built on the preceding developments and the very extensive RXTE data archive for BHBs. In Figure 2, we illustrate the character of each state by showing examples of PDSs and energy spectra for the BHB GRO J1655-40. The relevance of X-ray states fundamentally rests on the large differences in the energy spectra and PDSs that can be seen in a comparison of any two states. For thorough discussions, many illustrative spectra, and detailed references, see McClintock \& Remillard (2006). Discussions of physical models for these states are given in $\S 7$.

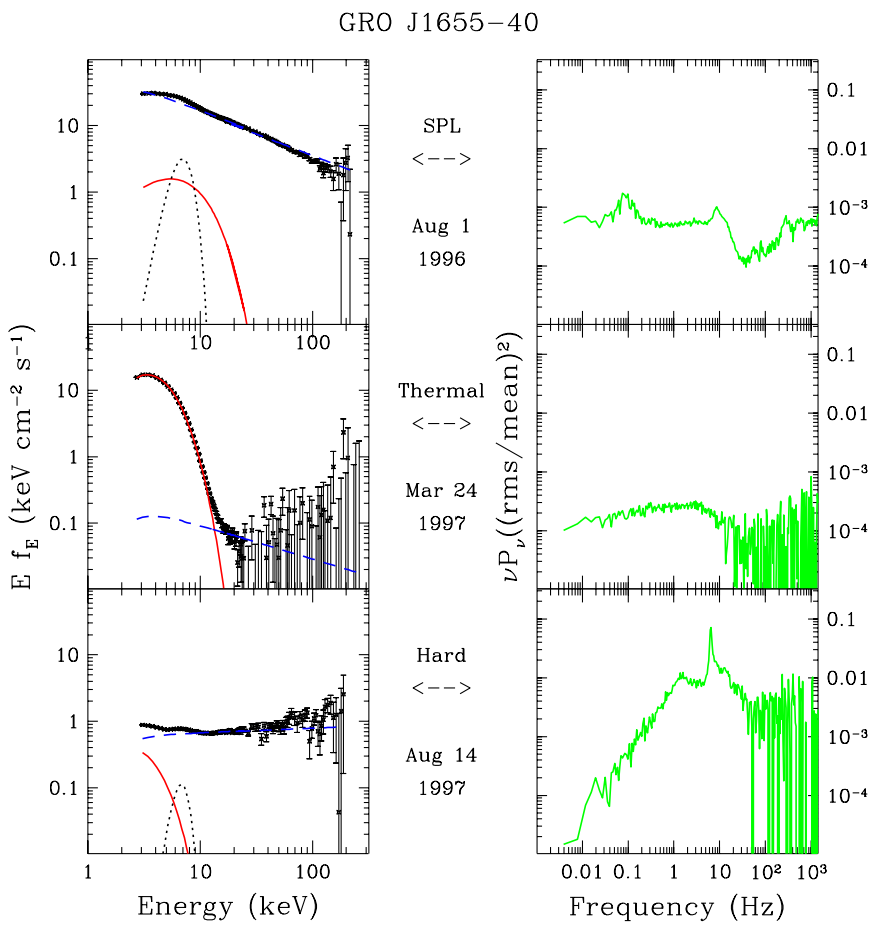

Figure 2: Sample spectra of black-hole binary GRO J1655-40 illustrating the three outburst states: steep power law, thermal, and hard. Each state is characterized by a pair of panels. Left panels show the spectral energy distribution decomposed into three components: thermal (red, solid line), power-law (blue, dashed line), and a relativistically broadened $\mathrm{Fe} \mathrm{K} \alpha$ line (black, dotted line). Right panels show the PDSs plotted as $\log \left(\nu \times P_{\nu}\right)$ versus $\log \nu$.

The sharpest point of departure from the old description of states is that luminosity is abandoned as a criterion for defining the state of a source. In defining states, McClintock \& Remillard (2006) adopted the pragmatic and generic strategy of utilizing a spectral model consisting of a multitemperature accretion disk and a PL component (with a possibile break near $15 \mathrm{keV}$ or an exponential cutoff in the range $30-100 \mathrm{keV}$ ). When required, an Fe emission line or a reflection component was included. The model also included photoelectric absorption by 
Table 2: Outburst states of black holes: nomenclature and definitions

\begin{tabular}{|c|c|}
\hline $\begin{array}{l}\text { New State Name } \\
\text { (Old State Name) }\end{array}$ & Definition of X-ray State ${ }^{a}$ \\
\hline $\begin{array}{l}\text { Thermal } \\
\text { (High/Soft) }\end{array}$ & $\begin{array}{l}\text { Disk fraction } f^{b}>75 \% \\
\text { QPOs absent or very weak: } a_{\max }^{c}<0.005 \\
\text { Power continuum level } r^{d}<0.075^{e}\end{array}$ \\
\hline $\begin{array}{l}\text { Hard } \\
\text { (Low/Hard) }\end{array}$ & $\begin{array}{l}\text { Disk fraction } f^{b}<20 \% \text { (i.e., Power-law fraction }>80 \% \text { ) } \\
1.4^{f}<\Gamma<2.1 \\
\text { Power continuum level } r^{d}>0.1\end{array}$ \\
\hline $\begin{array}{l}\text { Steep Power Law (SPL) } \\
\text { (Very high) }\end{array}$ & $\begin{array}{l}\text { Presence of power-law component with } \Gamma>2.4 \\
\text { Power continuum level } r^{d}<0.15 \\
\text { Either } f^{b}<0.8 \text { and } 0.1-30 \mathrm{~Hz} \text { QPOs present with } a^{c}>0.01 \\
\text { or disk fraction } f^{b}<50 \% \text { with no QPOs }\end{array}$ \\
\hline \multicolumn{2}{|c|}{$\begin{array}{l}{ }^{a} 2-20 \mathrm{keV} \text { band. } \\
{ }^{b} \text { Fraction of the total } 2-20 \mathrm{keV} \text { unabsorbed flux. } \\
{ }^{c} \text { QPO amplitude (rms). } \\
d^{d} \text { Total rms power integrated over } 0.1-10 \mathrm{~Hz} . \\
{ }^{e} \text { Formerly } 0.06 \text { in McClintock \& Remillard (2006). } \\
{ }^{f} \text { Formerly } 1.5 \text { in McClintock \& Remillard (2006). }\end{array}$} \\
\hline
\end{tabular}

neutral gas. McClintock \& Remillard (2006) used four parameters to define X-ray states: (1) the disk fraction $f$, which is the ratio of the disk flux to the total flux (both unabsorbed) at $2-20 \mathrm{keV} ;(2)$ the PL photon index $(\Gamma)$ at energies below any break or cutoff; (3) the rms power $(r)$ in the PDS integrated from $0.1-10 \mathrm{~Hz}$, expressed as a fraction of the average source count rate; and (4) the integrated rms amplitude $(a)$ of any QPO detected in the range 0.1-30 Hz. PDS criteria ( $a$ and $r$ ) utilize a broad energy range, e.g., the bandwidth of the RXTE PCA instrument, which is effectively $2-30 \mathrm{keV}$. Quantitative definitions of the three states are given in Table 2.

In the thermal state (formerly high/soft state, and "thermal dominant" state in McClintock \& Remillard 2006) the flux is dominated by the heat radiation from the inner accretion disk, the integrated power continuum is faint, and QPOs are absent or very weak (see Table 2). There is usually a second, nonthermal component in the spectrum, but its contribution is limited to $<25 \%$ of the flux at $2-20 \mathrm{keV}$. The state is illustrated in the middle row of panels in Figure 2. The spectral deconvolution shows that the thermal component (red line) is much

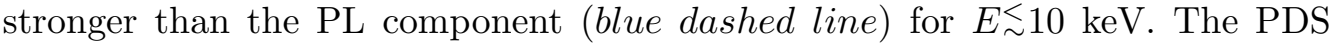
(right panel), which is plotted in terms of $\log \left(\nu \times P_{\nu}\right)$ versus $\log \nu$, appears featureless. Similar displays of paired energy spectra and PDSs for nine other BHBs/BHCs in the thermal state are shown in McClintock \& Remillard (2006).

The hard state (formerly low/hard state) is characterized by a hard PL component $(\Gamma \sim 1.7)$ that contributes $\geq 80 \%$ of the $2-20 \mathrm{keV}$ flux (Table 2$)$. The power continuum is bright with $r>0.1$ and QPOs may be either present or absent. A hard state osbervation of GRO J1655-40 is shown in the bottom row of panels in Figure 2. The accretion disk appears to be faint and cool compared to the thermal state. As noted previously ( $\S 4.1)$, the hard state is associated with the presence of a quasi-steady radio jet, and clear correlations between the radio and X-ray intensities are observed. 
The hallmark of the steep power law (SPL) state (formerly the very high state) is a strong $\mathrm{PL}$ component with $\Gamma \sim 2.5$. In some sources, this $\mathrm{PL}$ has been detected without a break to energies of $\sim 1 \mathrm{MeV}$ or higher $(\S 4.1)$. This state is also characterized by the presence of a sizable thermal component and the frequent presence of X-ray QPOs (see Table 2). An example of the SPL state is shown in the top row of panels in Figure 2, and many additional illustrations are displayed in McClintock \& Remillard (2006). There are similarities between the SPL state and the thermal state; both show a thermal component and a steep PL component. However in the thermal state the PL is faint and has a more variable photon index, while the SPL state is plainly distinguished by its powerful PL component and the commonly-occurring QPOs. The SPL state tends to dominate BHB spectra as the luminosity approaches the Eddington limit, and it is this state that is associated with high-frequency QPOs $(\S 6.2 ;$ McClintock \& Remillard 2006).

Intermediate states and state transitions are another important aspect of BHB studies. The three states defined by McClintock \& Remillard (2006) attempt to specify spectral and timing conditions that are quasi-stable and that appear to have distinct physical origins. There are gaps in the parameter ranges used to define these states (Table 2), and this gives rise to intermediate states. The hybrid of the hard and SPL states is particularly interesting for its correlations with radio properties $(\S 4.3$ and $\S 5)$ and also with disk properties $(\S 6.1)$.

\subsection{The Unified Model for Radio Jets}

Many researchers investigate the spectral evolution of BHBs using a hardnessintensity diagram (HID), which is a plot of X-ray intensity versus a "hardness ratio" $(H R)$, i.e., the ratio of detector counts in two energy bands (e.g., van der Klis 2005; Homan et al. 2001; Belloni 2004). This diagram is widely used in tracking the behavior of accreting neutron stars. Compared to the spectralfitting approach described above, the HID approach has the advantage that it is model independent and the disadvantage that it is difficult to relate the results to physical quantities. Interpretations of variations in the HID depend on the particular energy bands chosen to define $H R$ in a given study. If both bands are above $\sim 5 \mathrm{keV}$, then the $H R$ value effectively tracks the slope of the PL component (i.e., lower $H R$ is a steeper PL). Softer energy bands admit a mixture of thermal and nonthermal components, and interpretations are then more complicated.

The HID is also used in illustrating the "unified model for radio jets" proposed by Fender et al. (2004). Figure 3 shows their schematic for the relationships between jets and X-ray states, where the state of an observation is distinguished simply by the value of $H R$. The figure shows qualitatively how the jet Lorentz factor (lower panel) and the morphology of the jet (sketches $i-i v$ ) evolve with changes in the X-ray state. Tracks for state transitions of an X-ray source in the HID are also shown (top panel). The solid vertical line running through both panels in Figure 3 is the "jet line." To the right of the jet line the X-ray spectrum is relatively hard and a steady radio jet is present, and to the left the spectrum is soft and the jet is quenched. The jet line also marks an instability strip where violent ejections of matter may occur (see sketch iii), as indicated by the spike in the Lorentz factor (lower panel). The tracks for state evolution in the HID are influenced by observations of GX 339-4 (Belloni et al. 2005). In the Belloni et al. study, $H R$ was defined as the ratio of source counts at $6.3-10.5 \mathrm{keV}$ to the 


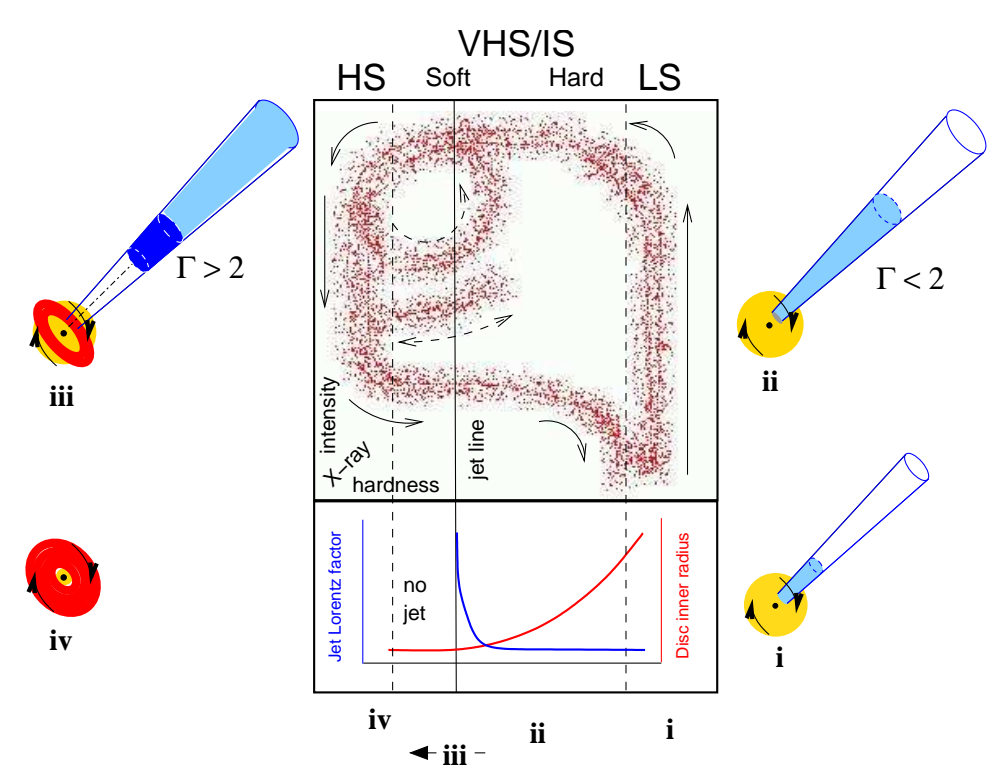

Figure 3: A schematic representation of the model for disk-jet coupling in blackhole binaries from Fender et al. (2004). The top panel shows evolutionary tracks in a HID, which is a plot of X-ray intensity versus X-ray hardness. (These quantities increase upward and to the right, respectively.) The bottom panel gives a qualitative impression of how the jet's bulk Lorentz factor (blue curve) and the inner disk radius (red curve) vary with X-ray hardness. The X-ray states are labeled at the top in the old nomenclature (i.e., HS = high/soft state; VHS/IS $=$ very high and intermediate states; and $\mathrm{LS}=$ low $/$ hard state.)

counts at $3.8-7.3 \mathrm{keV}$.

The vertical source track on the right side of Figure 3 corresponds to the low/hard or hard state with $H R>0.8$ for GX 339-4 (see Belloni it al. 2005). The vertical track on the far left (and most observations of GX 339-4 with $H R<$ $0.2)$ are in the high/soft or thermal state. Thus, the HID state classifications and the McClintock \& Remillard (2006) classifications agree very well in these two regimes. However, for the intermediate values of $H R$, which fall between the dashed lines, the states are described differently. The states in this region are further divided by Belloni et al. (2005) into "soft intermediate" and "hard intermediate" states, based on the $H R$ values and the properties of the PDS power continuum. This completes a description of the four X-ray states defined in the unified jet model. Further comparisons of the HID state classifications and the McClintock \& Remillard (2006) classifications are given in $\S 5$.

The unified model for X-ray states and radio jets provides opportunities to study the disk-jet coupling explicitly, and the HID format is very easy to apply to observations. On the other hand, the state definitions of McClintock \& Remillard (2006) are more quantitative, and they provide spectral information that is more directly applicable to physical models. In $\S 5$, we present outburst data for selected $\mathrm{BHB} / \mathrm{BHC}$ systems using both depictions for X-ray states. 


\subsection{Quiescent State}

The quiescent state corresponds to luminosities that are three or more orders of magnitude below the levels of the active states described above. The typical BHB with transient outbursts spends most of its time in a quiescent state that is characterized by an extraordinarily faint luminosity $\left(L_{x}=10^{30.5}-10^{33.5} \mathrm{erg} \mathrm{s}^{-1}\right)$ and a spectrum that is distinctly nonthermal and hard $(\Gamma=1.5-2.1)$. The quiescent state is particularly important in two ways: (a) It enables firm dynamical measurements to be made because the optical spectrum of the secondary star becomes prominent and is negligibly affected by X-ray heating (van Paradijs \& McClintock 1995); and (b) its inefficient radiation mechanism underpins a strong argument for the event horizon ( $(8.1)$.

For a thorough review of the X-ray properties of this state and discussions of physical models, see McClintock \& Remillard (2006). As commonly remarked, it is possible that the hard and quiescent states represent a single mechanism that operates over several orders of magnitude in X-ray luminosity. However, this question remains controversial. Corbel, Tomsick \& Kaaret (2006) have recently derived precise spectral parameters for XTE J1550-564. They conclude that the quiescent spectrum of this source (and a few others) is softer than the spectrum in the much more luminous hard state.

\section{X-ray Overview of State Evolution and Energetics}

\subsection{Overview Plots for Six Individual Sources}

In $\S 4$, we described two ways of defining X-ray states: a quantitative method based on generic X-ray spectral modeling and PDS analysis (McClintock \& Remillard 2006), and another based on radio properties, X-ray PDSs, and HIDs. Here we synthesize the two approaches to provide the reader with a comprehensive picture of the behavior of an accreting $\mathrm{BH}$.

In Figures 4-9 respectively, we show detailed overviews of the behavior of six X-ray novae: five BHBs and one BHC (H 1743-322). Each figure contains seven panels that review the data for a single source. The overview plots can be used in two ways. One can focus on the figure (7 panels) for a particular BHB and examine for each color-coded state many aspects of the behavior of the source. The key science questions addressed by each panel are: $(a, b)$ How do states and luminosity vary with time?; $(c)$ How does the radiation energy divide between thermal and nonthermal components $(2-20 \mathrm{keV})$ ?; $(d)$ How do the states of McClintock and Remillard (2006) relate to the states of Fender, Belloni, and Gallo (2004) which are presented in a HID?; and $(e, f, g)$ How do three key Xray properties - PL index, disk fraction, and rms power - vary as a function of either the hardness ratio or the X-ray state? On the other hand, one can choose a particular panel and compare the behavior of the six sources to draw general conclusions about common behavior patterns in BHBs as well as their differences. Such conclusions are discussed at the end of this Section and in $\S 5.2$.

The following comments pertain to all six figures. As an inspection of the various ASM light curves (panel a) shows, the data cover outbursts observed with RXTE during the time interval 1996-2004. The remaining panels $(b-g)$ show results derived from RXTE pointed observations. These latter panels display several different kinds of data, but in every panel there is a common use of 
symbol type and color to denote the state of the source (McClintock \& Remillard 2006 definitions): thermal (red $x$ ), hard (blue square), SPL (green triangle), and any intermediate type (black circle).

Panel $b$, which mimics the ASM light curve, shows the $2-20 \mathrm{keV}$ X-ray flux derived from the fitted spectral model (see §3.3). Panel $c$, which is also based on the fitting results, shows how the energy is divided between the thermal (accretion disk) component and the nonthermal (PL) component; we refer to this plot as the energy-division diagram. Panel $d$, which is based on raw count rates, shows how the states are distributed in the hardness intensity diagram (HID). We use this panel to examine how the McClintock \& Remillard (2006) states are distributed in the HID and how they correspond to the states that are defined by the unified jet model (§4.3). Finally, in panels $e-g$ we plot spectral hardness on the x-axis versus three of the parameters that are used to define BH states, namely, the PL index $\Gamma$, the disk fraction, and the integrated rms power in the PDS (0.1$10 \mathrm{~Hz})$. The HIDs use the normalization scheme and hard color definition $(H R$ $=8.6-18.0 \mathrm{keV}$ counts / 5.0-8.6 keV counts) given by Muno et al. (2002), for which the Crab Nebula yields $2500 \mathrm{c} \mathrm{s}^{-1} \mathrm{PCU}^{-1}$ and $H R=0.68$. The RXTE pointed observations are selected to have a minimum exposure Rtime of $500 \mathrm{~s}$ and a minimum source flux of $10^{-10} \mathrm{erg} \mathrm{cm}^{-2} \mathrm{~s}^{-1}$ (i.e., 4 mCrab) at $2-20 \mathrm{keV}$.

We begin our discussion of individual sources with GRO J1655-40, the BHB used in $\S 4.2$ to illustrate the BH states (McClintock \& Remillard 2006). In Figure 4 we show the overview for the 1996-1997 outburst. The data are mostly derived from publications (Sobczak et al. 1999; Remillard et al. 1999; 2002a), with some supplementary results added for completeness. GRO J1655-40 shows an orderly and monotonic evolution of states along the arcs displayed in the energydivision diagram (panel c) and the HID (panel d). In the HID, the McClintock \& Remillard (2006) states are cleanly sorted along the hardness axis. Furthermore, panels $e-g$ show clear correlations between the key state parameters and the hardness ratio. Quite similar behavior is seen in the X-ray overview for the next BHB, 4U 1543-47 (Figure 5). Here we have used the results derived by Park et al (2004), while adding results from similar analyses to extend the coverage to the end of the outburst. As these two overviews show (i.e., Figures 5 and 6), both sources favor the thermal state, and they only enter the hard state at low luminosity.

Figure 6 exhibits four outbursts of XTE J1550-564 with successively shorter durations and decreasing maxima (panels $a$ and $b$ ). The results shown in panels $c-g$ are dominated by the first outburst (1998-1999), which provides 202 of 309 state assignments and most of the points at high flux levels. Several authors have noted the complex behavior of this source (e.g., Sobczak et al. 2000b; Homan et al. 2001). The energy-division diagram (panel c) and the HID (panel $d$ ) display many branches. The thermal branch (red $x$ symbols) in the energy diagram covers a wide range of luminosity. Note that there are examples of both high luminosity hard-state observations and low luminosity SPL-state observations. One must conclude that luminosity does not drive a simple progression of X-ray states (as implied by the old state names: low/hard, high/soft and very high). Thus, the X-ray state must depend on some unknown and important variable(s) in addition to the BH mass and the mass accretion rate (Homan et al. 2001).

Similar patterns of behavior are shown in the next two examples. The overview for $\mathrm{H}$ 1743-322 (Figure 7, panels $b-g$ ) shows many state transitions (panel $b$ ), and the complex tracks in panels $c$ and $d$ are reminiscent of XTE J1550-564 


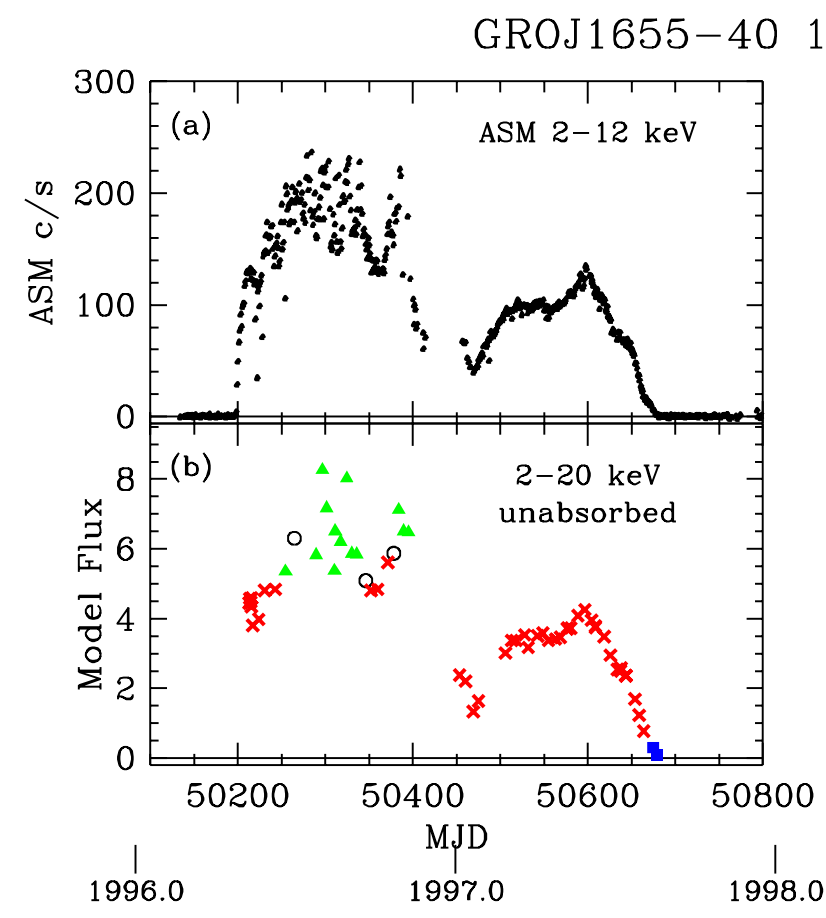

$1996-1997$
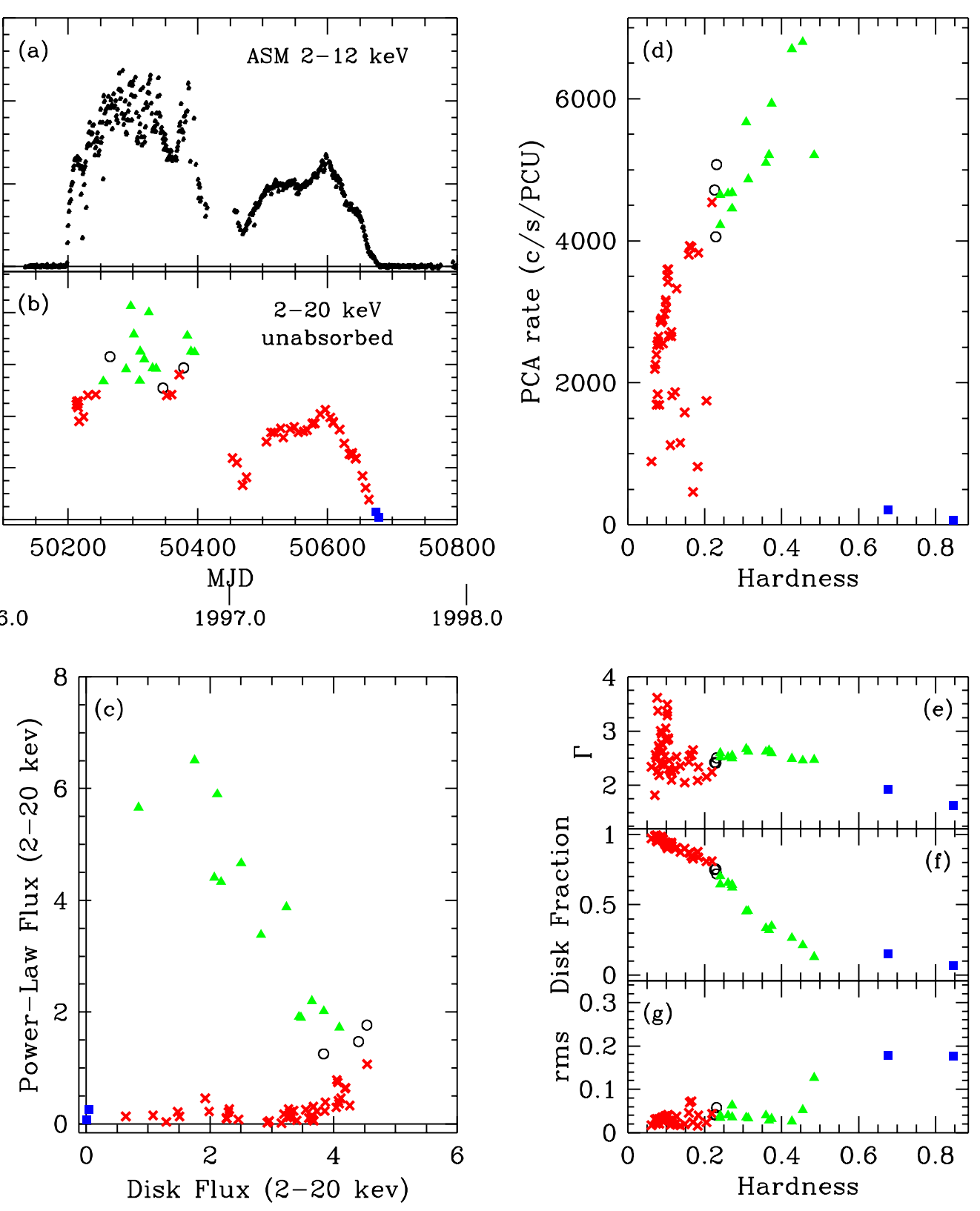

Figure 4: X-ray overview of GRO J1655-40 during its 1996-1997 outburst. The ASM light curve is shown in panel $a$. All other data are derived from RXTE pointed observations binned in 62 intervals. The symbol color and type denote the X-ray stat: thermal (red $x$ ), steep power law (SPL) (green triangle), hard (blue square), and any intermediate type (black circle). This outburst shows simple patterns of evolution that favor the thermal and SPL states.

(Figure 6). The hysteresis in transitions to and from the hard state are especially evident in the HID (panel d; see also Maccarone \& Coppi 2003). The overview 

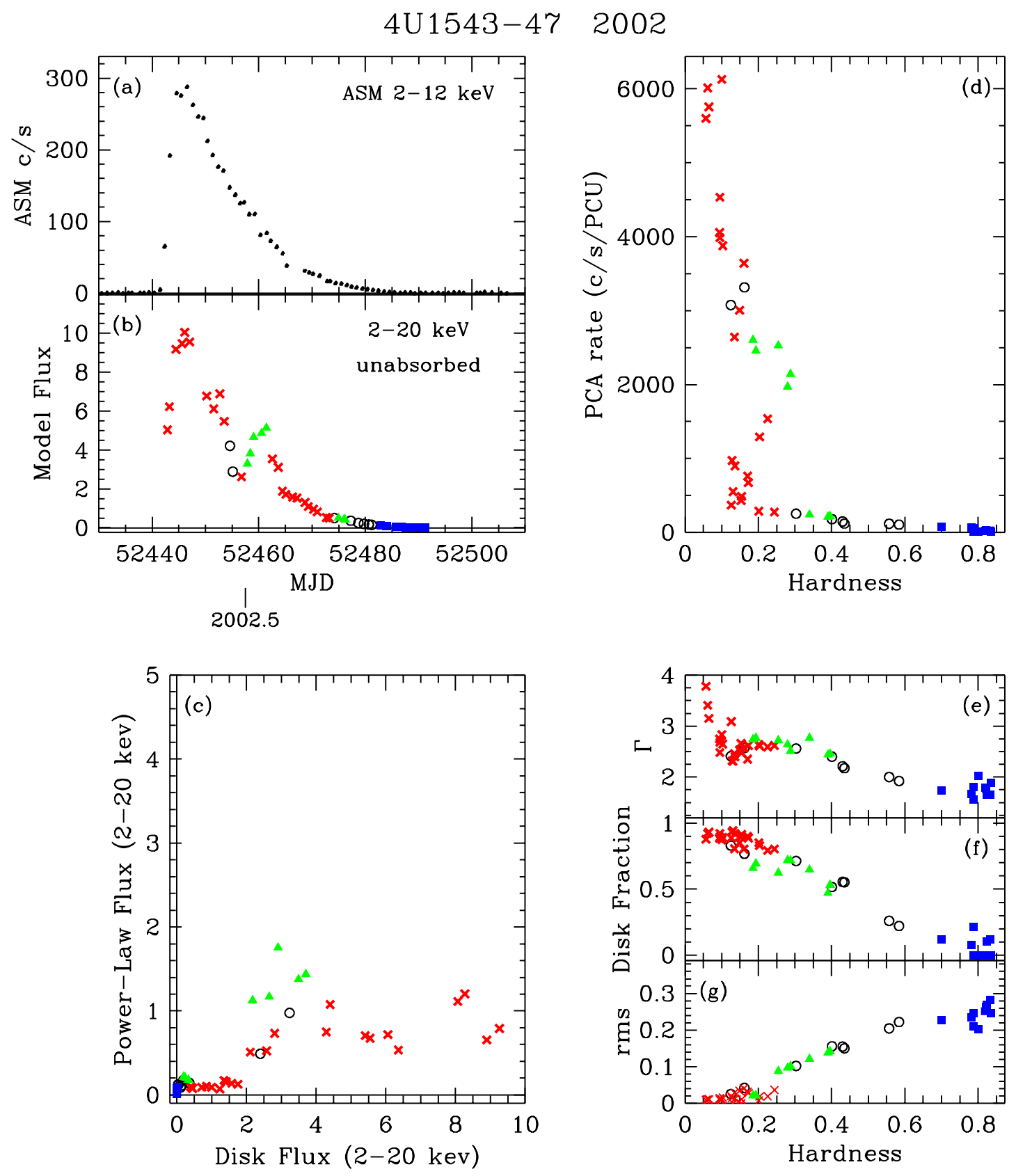

Figure 5: X-ray overview of $4 \mathrm{U}$ 1543-47 during its 2002 outburst. The presentation format and the state-coded plotting symbols follow the conventions of Figure 4. The RXTE pointed observations (49 time intervals) show relatively simple patterns of state evolution, and the thermal state is prevalent when the source is bright.

for XTE J1859+226 (Figure 8), is similar to that of $\mathrm{H} \mathrm{1743-322.} \mathrm{All} \mathrm{of} \mathrm{the}$ brightest observations are found in the SPL state, and the vertical track in the HID (green triangles in Figure $8 d$ ) is a consequence of constant values of $\Gamma$ 
in the SPL state. These complex results reinforce the need to probe deeply in attempting to identify the underlying variables that govern the emission of radiation from BHBs.
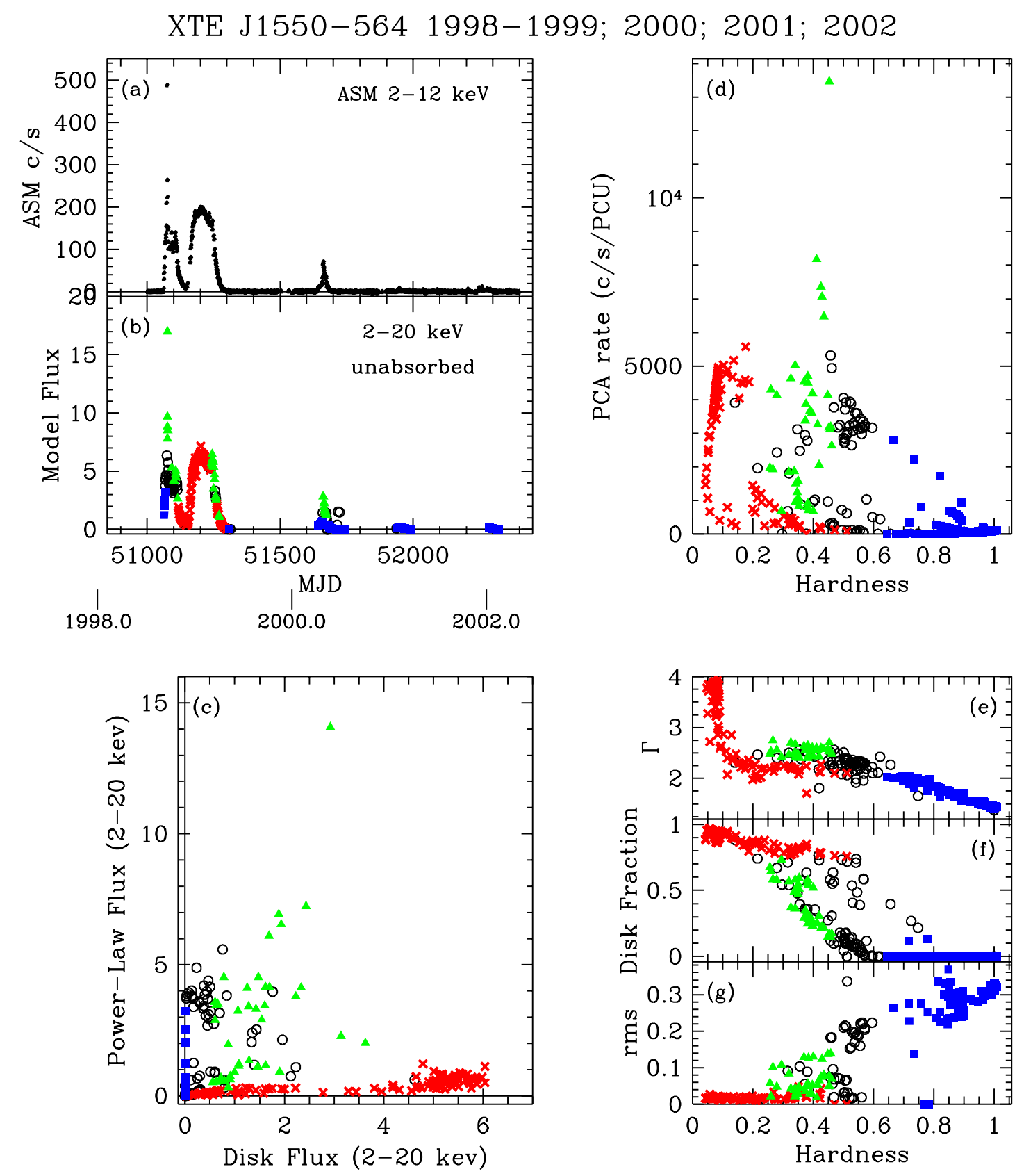

Figure 6: X-ray overview (309 time intervals) of XTE J1550-564 that includes a series of four outbursts with decreasing maxima. The presentation format and the state-coded plotting symbols follow the conventions of Figure 4. The two brighter outbursts (1998-1999 and 2000) show great complexity in the temporal evolution of states and the energy division between thermal and nonthermal components. In contrast, the subsequent pair of faint outbursts were confined to the hard state. 
H1743-322
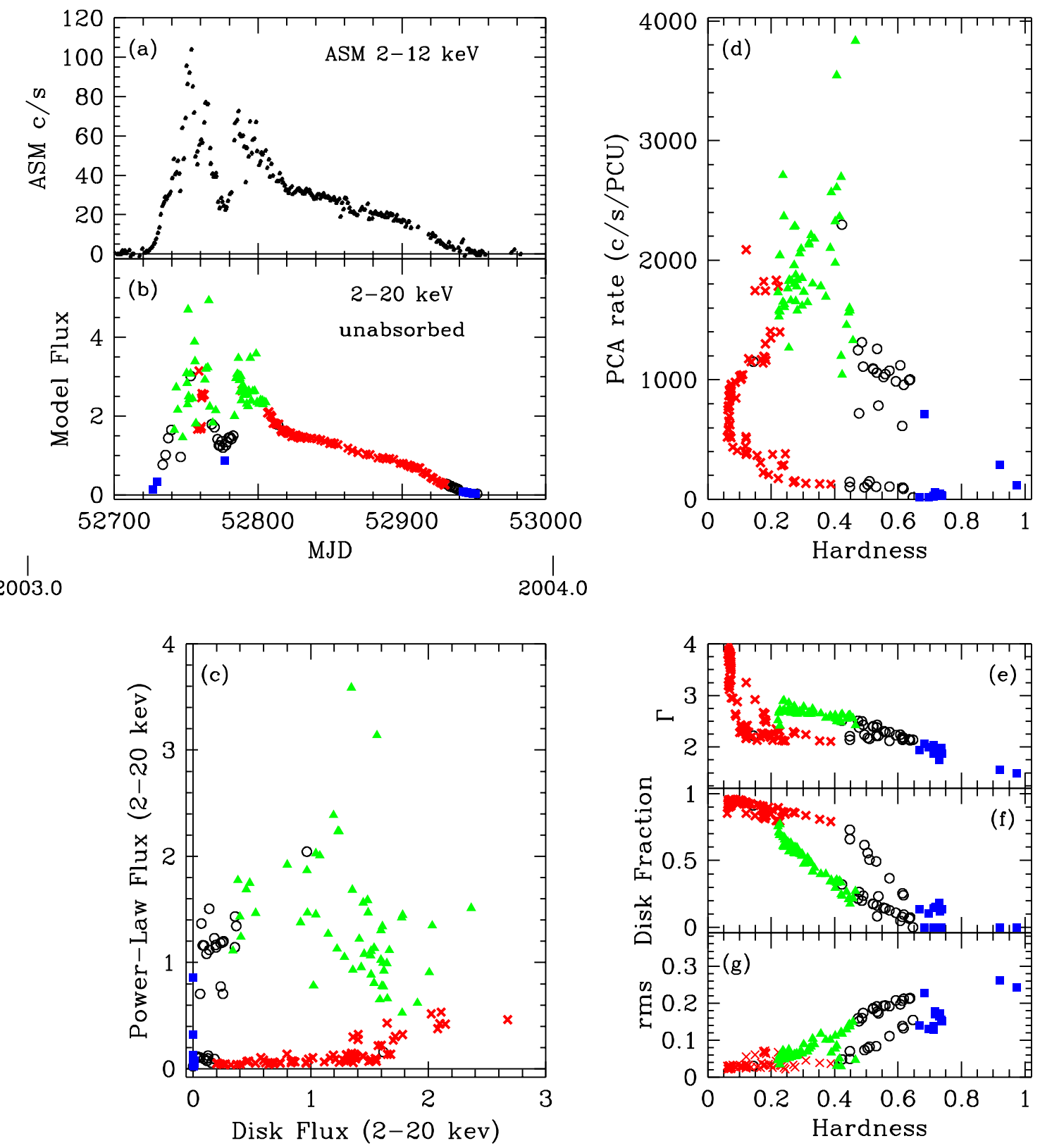

Figure 7: X-ray overview of H 1743-322 during its 2003 outburst. The presentation format and the state-coded plotting symbols follow the conventions of Figure 4. The RXTE pointed observations (170 time intervals) show complex state evolution with similarities to XTE J1550-564.

Finally, we show the overview of GX 339-4 in Figure 9. This source is known to produce frequent X-ray outbursts and to remain for long intervals in the hard state, as shown in panel $b$. It is therefore a frequent target for radio studies (e.g., Fender et al. 1999; Corbel et al. 2000; Gallo et al. 2003; Belloni et al. 2005). There is an apparent similarity between the HID for GX 339-4 (panel $d$ ) and 
XTE J1859+226 1999-2000
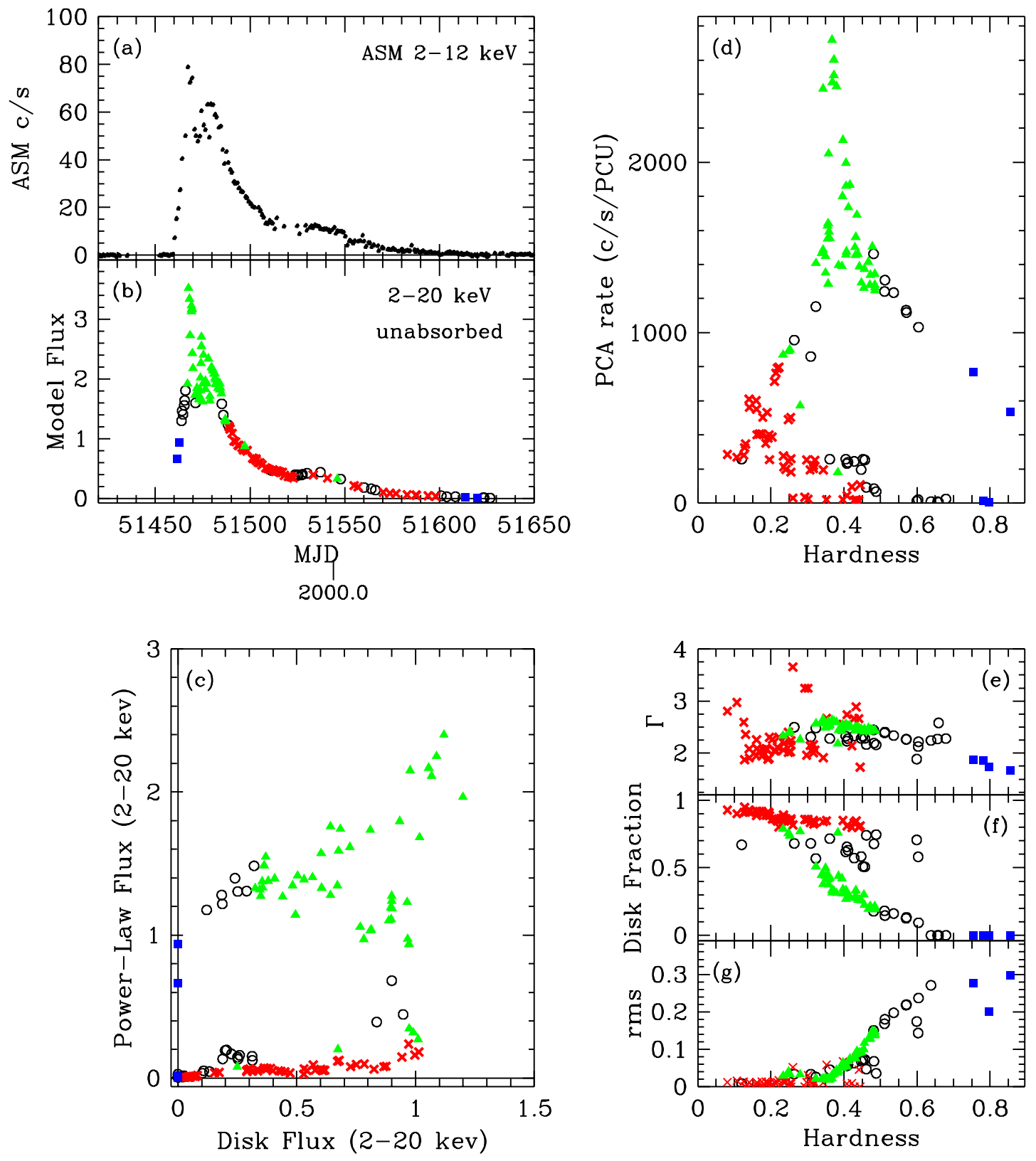

Figure 8: X-ray overview of XTE J1859+226 during its 1999-2000 outburst. The presentation format and the state-coded plotting symbols follow the conventions of Figure 4. The RXTE pointed observations (130 time intervals) show complex behavior very similar to that seen for $\mathrm{H}$ 1743-322. In panels $b, c$, and $d$ it is apparent that the steep power law state is prevalent when the source is bright.

to the schematic sketch in Figure 3 that illustrates the unified model for jets. The HID shows four branches for the hard state, one of which peaks at $75 \%$ of the maximum count rate in the thermal and SPL states. Despite the wide 
GX339-4 1996-2005
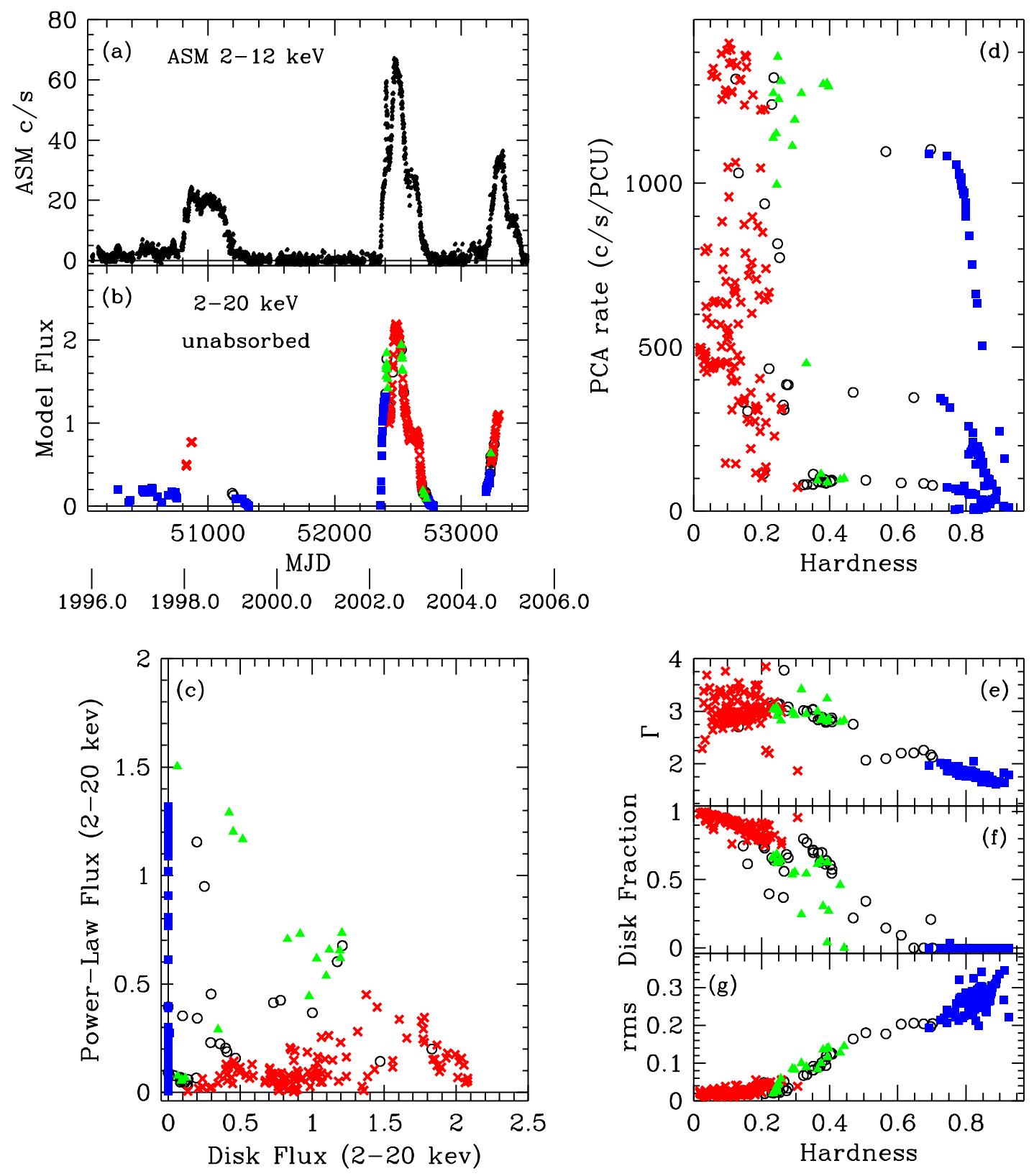

Figure 9: X-ray overview of GX 339-4 (274 time intervals). This source exhibits frequent outbursts and long intervals in the hard state at low luminosity. The presentation format and the state-coded plotting symbols follow the conventions of Figure 4. The state evolution shown here was used as the prototype for making the connection between the inner disk and a steady radio jet (compare panel $d$ and Figure 3).

range in luminosity for each of the three states, the plots of the key parameters (panels $e-g$ ) appear orderly and well correlated with the hardness ratio. Thus, 
the behavior of GX 339-4 supports the theme that recurs throughout this work, namely, that X-ray states are important, although they are plainly not a simple function of source luminosity.

The six X-ray overviews presented in Figures 4-9 show that BHB outbursts can be very complex. They typically begin and end in the hard state, but between those times there is common disorder in the temporal evolution of luminosity $(b$ panels), and in the division of radiation energy between thermal and nonthermal states ( $c$ panels). On the other hand, there are clear correlations involving the key spectral and timing properties, examined versus either $H R$ value or the colorcoded state symbols ( $e, f$, and $g$ panels). These results, which are robust for a variety of $\mathrm{BHBs}$, including sources displaying multiple outbursts, confirm the prevailing wisdom that complex BHB behavior can be productively organized and studied within the framework of X-ray states. Further discussions of the overview figures are continued in the following section.

\subsection{Conclusions from Overviews of X-ray States}

Several conclusions can be drawn from the set of overview plots shown in $§ 5$. First, when the state assignments of McClintock \& Remillard (2006) are examined in the HID format (i.e., sorting symbol color/type vs. hardness in the $d$ panels of Figures 4-9), it is apparent that these states and the designs of the unified-jet model overlap significantly. For example, for the particular $H R$ defined in $\S 5.1$, observations with $H R<0.2$ would be assigned to the high/soft state in the unified-jet model, and nearly all of these same observations are here assigned to the thermal state of McClintock \& Remillard (2006). Similarly, observations with $H R>0.65$ correspond to the hard state in both prescriptions. For the intermediate states in the HID, there is a divergence between the two approaches, and this is especially obvious for sources that show complex behavior, e.g., XTE J1550-564. For this source, the McClintock \& Remillard (2006) states are strongly disordered in the interval $0.20<H R<0.65$ (see Figure 6 panel $d$ ).

We have emphasized that source luminosity is not a criterion used for identifying X-ray states in either prescription ( $\$ 4.2$ and $\S 4.3)$. This is evident in Figures 4-9, because there are lines of constant flux that intercept several different McClintock \& Remillard (2006) states ( $b$ panels), and because there are lines of constant intensity that intercept observations with very different $H R$ values $(d$ panels). However, it is clear that there is still some degree of correlation between $\mathrm{X}$-ray states and source brightness. For example, all of the sources show transitions to the hard state when the source becomes faint during its decay phase (see the $b$ and $d$ panels in Figures 4-9). At the opposite extreme, the highest luminosity observations for four of the six sources occur in the SPL state (Figures $4 \& 6-8)$.

The energy-division diagrams $(2-20 \mathrm{keV}$; $c$ panels of Figures 4-9) routinely show vertical tracks for the hard state (blue squares), whereas horizontal tracks with gentle curvature are seen for the thermal state (red $x$ symbols). These tracks indicate a free flow of energy into the hard PL spectrum during the hard state and into the accretion disk during the thermal state. This point was first made by Muno et al. (1999) in describing the behavior of GRS 1915+105. The curvature of the thermal tracks ( $c$ panels, Figures 4-9) can be interpreted as being due to increased Comptonization with increasing thermal luminosity. Tracing smooth lines through these tracks would constrain Comptonization to a maximum of 
$<20 \%$ of the flux $(2-20 \mathrm{keV})$ at the peak of the thermal state. The energydivision tracks for the SPL state (green triangles) are not well defined, and the tracks of different sources do not resemble each other. The energy-division diagrams are more effective than the HIDs in delineating intrinsic differences between the SPL and thermal states.

Comparisons between the SPL and thermal states in terms of the photon index $(\Gamma ; e$ panels of Figures 4-9) are of interest, as these states are sometimes presumed to share a common PL mechanism that is relatively muted during the thermal state. Generally, we find broad distributions in $\Gamma$ during the thermal state, and in some cases the thermal tracks are shifted from the SPL tracks (e.g., Figures $6 e, 7 e, 8 e)$. We find no compelling evidence that the PL mechanisms for the thermal and SPL states are the same.

In the following section, we consider one additional observational topic - X-ray QPOs - before discussing the ongoing efforts to relate X-ray states to physical models for $\mathrm{BH}$ accretion.

\section{$6 \quad$ X-ray Quasi-Periodic Oscillations}

X-ray QPOs are specialized and extraordinarily important avenues for the study of accreting BHs. They are transient phenomena associated with the nonthermal states and state transitions. For definitions of QPOs and analysis techniques, see van der Klis (2005) and some details in $§ 3.2$.

QPOs play an essential role in several key science areas, such as probing regions of strong field ( $\$ 6.2 \& \$ 8.2 .4)$ and defining the physical processes that distinguish $\mathrm{X}$-ray states. Thus far in this review, however, QPOs have been considered only tangentially (e.g., §4.2). In this section we focus on the significance of QPOs, their subtypes, and spectral/temporal correlation studies that involve the QPO frequency. Following the literature, we divide the discussions of QPOs into lowfrequency and high-frequency groups. In doing so, we disregard the additional, infrequent appearances of very low frequency QPOs (e.g., the QPO below $0.1 \mathrm{~Hz}$ in the upper-right panel of Figure 2) that are not understood. Physical models for QPOs are briefly discussed in $\S 7$, and the importance of high-frequency QPOs for probing strong gravity is highlighted in $\S 8.2 .4$.

\subsection{Low-Frequency Quasi-Periodic Oscillations}

Low-frequency QPOs (LFQPOs; roughly 0.1-30 Hz)) have been detected on one or more occasions for 14 of the 18 BHBs considered in Table 4.2 of McClintock \& Remillard (2006). They are important for several reasons. LFQPOs can have high amplitude (integrated $\mathrm{rms} /$ mean values of $a>0.15$ ) and high coherence (often $Q>10$ ), and their frequencies and amplitudes are generally correlated with the spectral parameters for both the thermal and PL components (e.g., Muno et al. 1999; Sobczak et al. 2000a; Revnivtsev et al. 2000; Vignarca et al. 2003). With the exception of Cyg X-1, QPOs generally appear whenever the SPL contributes more than $20 \%$ of the flux at $2-20 \mathrm{keV}$ (Sobczak et al. 2000a), which is one component of the definition of the SPL state (Table 2; McClintock \& Remillard 2006).

LFQPOs can vary in frequency on timescales as short as one minute (e.g., Morgan et al. 1997). On the other hand, LFQPOs can also remain relatively stable and persistent. For example, in the case of GRS 1915+105, a 2.0-4.5 Hz 
QPO is evident in every one of the 30 RXTE observations conducted over a 6month interval in 1996-1997 (Muno et al. 2001). This degree of stability suggests that LFQPOs are tied to the flow of matter in the accretion disk. However, the frequencies of these QPOs are much lower than the Keplerian frequencies for the inner disk. For example, a $3 \mathrm{~Hz}$ orbital frequency around a Schwarzschild $\mathrm{BH}$ of $10 M_{\odot}$ corresponds to a radius near $100 R_{\mathrm{g}}$, while the expected range of radii for $\mathrm{X}$-ray emission in the accretion disk is $\lesssim 10 R_{\mathrm{g}}$.

LFQPOs are seen in the SPL state, the hard:SPL intermediate state, and in some hard states, particularly when the X-ray luminosity is high (McClintock \& Remillard 2006; Rossi et al. 2004). The rms amplitude generally peaks at photon energies $\gtrsim 10 \mathrm{keV}$ (e.g., Vignarca et al. 2003; Rodriguez et al. 2002), and detections have been made at energies above $60 \mathrm{keV}$ (Tomsick \& Kaaret 2001). This behavior clearly ties LFQPOs to the nonthermal component of the $\mathrm{X}$-ray spectrum. However, in principle, the oscillation could still originate in the accretion disk if the PL mechanism is inverse Compton scattering of disk photons and the coherence of the original oscillation is not destroyed by the scattering geometry. Alternatively, the mechanism that creates the energetic electrons required for Comptonization could be an oscillatory type of instability. In this case, the disk temperature or the thermal energy flux might control the QPO properties.

These concepts regarding QPO origins motivate correlation studies that compare QPO frequencies and amplitudes with various spectral parameters. In Figure 10 we show an example of such a correlation between LFQPO frequency and disk flux for two sources. The X-ray states are symbol coded, using the conventions adopted in $\S 5$. The QPO frequencies in the hard and intermediate states are highly correlated with disk flux, but this is not true for the QPOs at higher frequency in the SPL state. Presently, there is no explanation for this result. Possibly, the QPO mechanisms could differ between the hard and SPL states, or there could be a common mechanism that exhibits some type of dynamical saturation as the source moves into the SPL state.

Another avenue for QPO investigations is the study of phase lags and coherence functions that compare two different energy bands, e.g., 2-6 versus 13-30 $\mathrm{keV}$. Such analyses have been conducted for the QPOs in XTE J1550-564. Unexpectedly, the phase lag measurements showed groups distinguished by positive, negative, and zero lags defining, respectively, LFQPO types A, B, and C (Wijnands et al. 1999; Cui et al. 2000b; Remillard et al. 2002b; Casella et al. 2004). These details are surely complicated, but the ramifications are very significant. The A and B types are associated with the SPL state and the presence of highfrequency QPOs. On the other hand, C-type LFQPOs mostly occur in the intermediate and hard states, and they are responsible for the frequency versus disk flux correlation shown in Figure 10. There are also similarites between these BH LFQPO subtypes and the three QPOs of Z-type neutron-star systems (Casella, Belloni, \& Stella 2005).

Given the relatively high amplitudes of all LFQPOs above $6 \mathrm{keV}$, it is clear that the C-type oscillations are well connected to both the thermal and PL components in the X-ray spectrum. In this sense, QPOs can provide insights regarding the origin of the PL spectrum. Futhermore, we now have a comprehensive archive of accurate LFQPO measurements for a wide range of disk conditions. These data are available for testing any detailed models that are proposed to explain the nonthermal states in BHBs. 

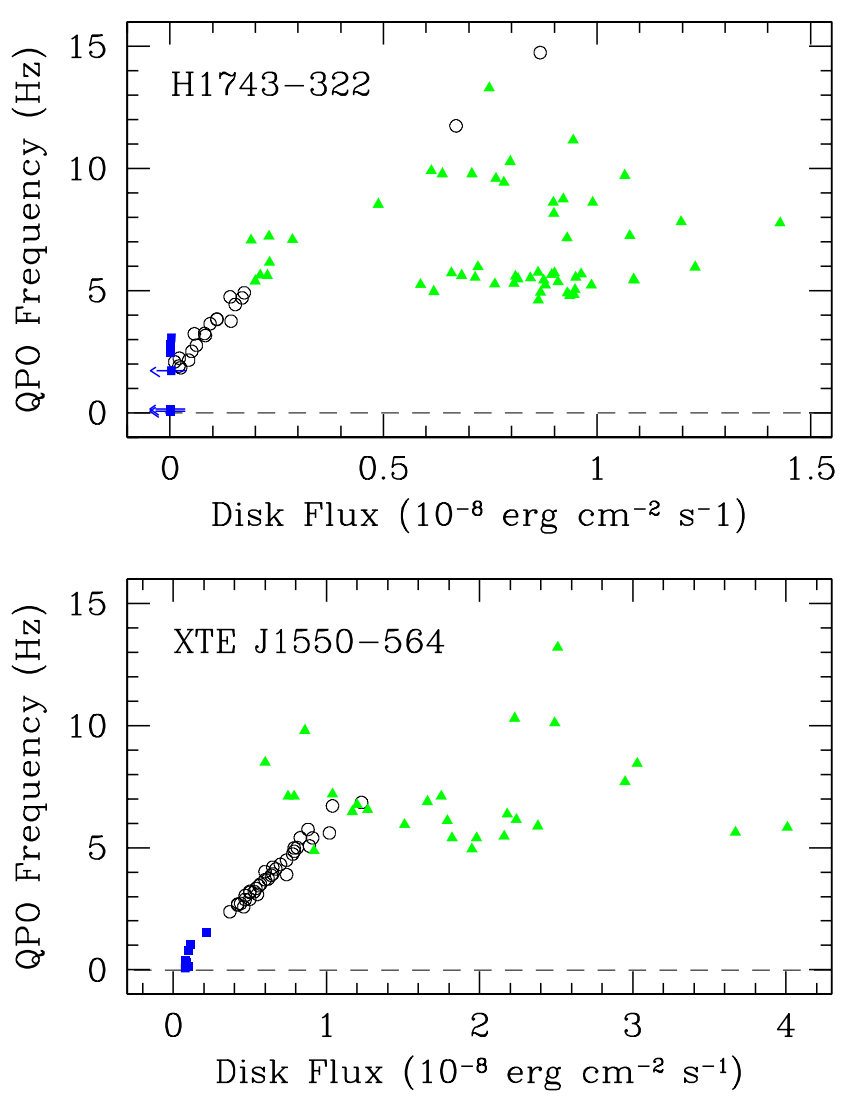

Figure 10: Quasi-periodic oscillation (QPO) frequency versus disk flux for XTE J1550-564 and H 1743-322. See Figure 4 for definitions of the symbol types. The QPO frequencies in the hard and especially the hard:SPL intermediate state are highly correlated with disk flux; these are C-type low-frequency QPOs (LFQPOs) based on their phase lags (see text). The steep power law QPOs are not correlated with disk flux, and these are A and B type LFQPOs.

\subsection{High-Frequency Quasi-Periodic Oscillations}

High-frequency QPOs (HFQPOs; 40-450 Hz) have been detected in seven sources (5 BHBs and 2 BHCs). These oscillations are transient and subtle $(a \sim 0.01)$, and they attract interest primarily because their frequencies are in the expected range for matter in orbit near the ISCO for $\mathrm{a} \sim 10 M_{\odot} \mathrm{BH}$.

As an aside, we briefly note that broad power peaks $(Q<1)$ have been reported at high frequencies in a few cases (e.g., Cui et al 2000a; Homan et al. 2003; Klein-Wolt, Homan, \& van der Klis 2004). At the present time, these broad PDS features do not impact the field significantly, because they are relatively rare and poorly understood. Consequently, we do not consider these broad features further.

The entire sample of HFQPOs with strong detections $(>4 \sigma)$ is shown in Figure 11. Three sources have exhibited single oscillations (Cui et al. 2000a; Homan 


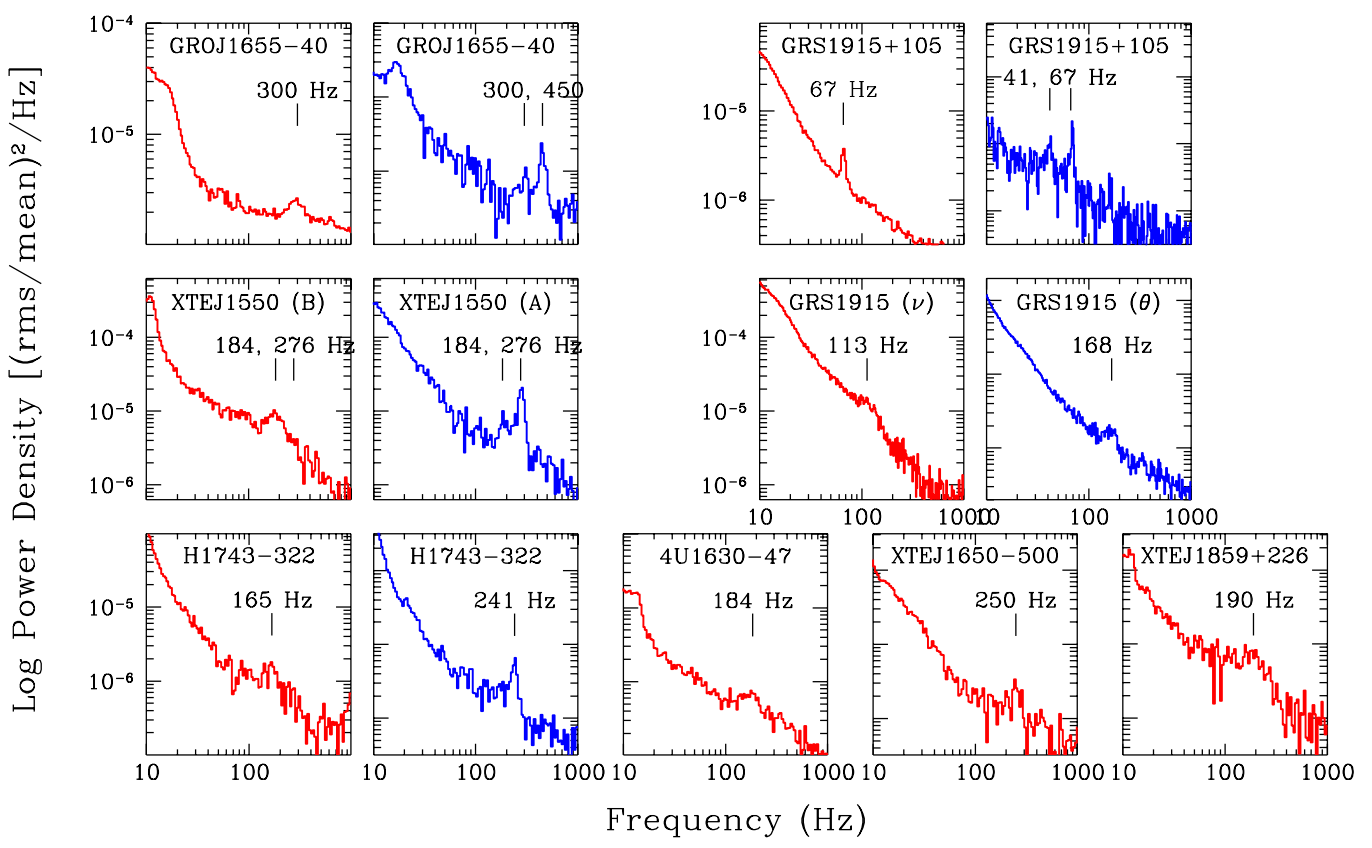

Figure 11: High-frequency quasi-periodic oscillations (HFQPOs) observed in black-hole binary and black-hole candidate systems. The traces in blue show power density spectra (PDSs) for the range 13-30 keV. Red traces indicate PDSs with a broader energy range, which may be either $2-30$ or $6-30 \mathrm{keV}$.

et al. 2003; Remillard 2004). The other four sources display pairs of HFQPOs with frequencies that scale in a 3:2 ratio. Most often, these pairs of QPOs are not detected simultaneously. The four sources are GRO J1655-40 (300, $450 \mathrm{~Hz}$; Remillard et al. 1999; 2002a; Strohmayer 2001a; Homan et al. 2005a), XTE J1550-564 (184, $276 \mathrm{~Hz}$; Remillard et al. 2002a; Homan et al. 2001; Miller et al. 2001), GRS 1915+105 (113, 168 Hz; Remillard 2004), and H 1743-322 (165, $241 \mathrm{~Hz}$; Homan et al. 2005b; Remillard et al. 2006b). GRS 1915+105 also has a second pair of HFQPOs with frequencies that are not in a 3:2 ratio (41, $67 \mathrm{~Hz}$; Morgan et al. 1997; Strohmayer 2001b).

HFQPOs are of further interest because they do not shift freely in frequency in response to sizable luminosity changes (factors of 3-4; Remillard et al. 2002a; 2006b). There is evidence of frequency shifts in the HFQPO at lower frequency (refering to the 3:2 pairing), but such variations are limited to 15\% (Remillard et al. 2002a; Homan et al. 2005a). This is an important difference between these BHB HFQPOs and the variable-frequency $\mathrm{kHz}$ QPOs seen in accreting neutron stars, where both peaks can shift in frequency by a factor of two (van der Klis 2005). Overall, BHB HFQPOs appear to be a stable and identifying "voice-print" that may depend only on the mass and spin of the $\mathrm{BH}(\S 8.2 .4)$.

All of the strong detections $(>4 \sigma)$ above $100 \mathrm{~Hz}$ occur in the SPL state. In three of the sources that exhibit HFQPOs with a $3: 2$ frequency ratio, the $2 \nu_{0}$ QPO appears when the PL flux is very strong, whereas $3 \nu_{0}$ appears when the PL flux is weaker (Remillard et al. 2002a; 2005b). Currently, there is no explanation for this result.

The commensurate frequencies of HFQPOs suggests that these oscillations are 
driven by some type of resonance condition. Abramowicz and Kluzniak (2001) proposed that orbiting blobs of accreting matter could generate the harmonic frequencies via a resonance between a pair of the coordinate frequencies given by GR. Earlier work had used GR coordinate frequencies and associated beat frequencies to explain fast QPOs in both neutron-star and BH systems (Stella et al. 1999), but without invoking a resonance condition. Current work on resonances as a means of explaining HFQPOs includes more realistic models for fluid flow in the Kerr metric. Resonance models are considered in more detail in $\S 8.2 .4$.

\section{Physical Models for X-ray States}

We briefly describe some physical models for the three active emission states $(\S 4)$ and QPO phenomena (§6). Our focus is on basic principles and the current interface between observations and theory.

\subsection{Thermal State}

For the thermal state there is a satisfactory paradigm, namely, thermal emission from the inner regions of an accretion disk. Observations and magnetohydrodynamic (MHD) simulations continue to increase our understanding of accretion disks. Also, fully-relativistic models of disk spectra have recently become publicly available, and results from this advance are discussed below.

The best-known hydrodynamic model of a radiating gas orbiting in the gravitational potential of a compact object is the steady-state, thin accretion disk model (Shakura \& Sunyaev 1973; Pringle 1981). A central problem for this model is a prescription for the viscosity that is required to drive matter inward and heat it, while transporting angular momentum outward. Initially, the viscosity was modeled using an ad hoc scaling assumption (Shakura \& Sunyaev 1973). This model leads to a temperature profile $T(R) \propto R^{-3 / 4}$ and the conclusion that the inner annulus in the disk dominates the thermal spectrum, because $2 \pi R d R \sigma T^{4} \propto L(R) \propto R^{-2}$. This result has a striking observational consequence: X-ray astronomy is the window of choice for probing strong gravity near the horizon of an accreting stellar-mass $\mathrm{BH}$.

The cardinal importance of the inner disk region highlights the need for an accurate model for the radiation emitted near the inner disk boundary associated with the ISCO (see $\S 1$ ). The ISCO lies at $6 R_{\mathrm{g}}$ for a Schwarzschild BH $\left(a_{*}=0\right)$, decreasing toward $1 R_{\mathrm{g}}$ as $a_{*}$ approaches 1 . Observationally, the thermal-state spectra of BHBs are well fitted using the classical model for a multitemperature accretion disk (Mitsuda et al. 1984; Makishima et al. 1986; Kubota \& Makishima 2004; Kubota et al. 2005). However, the derived spectral parameters (i.e., the temperature and radius of the inner disk) cannot be interpreted literally for several reasons. The model neglects the physically-motivated torque-free boundary condition at the ISCO (see Gierlinski et al. 2001; Zimmerman et al. 2005). Furthermore, the classical model ignores the sizable effects due to GR and radiative transfer (e.g., see Zhang, Cui, \& Chen 1997). Fortunately, accretion disk models for Kerr BHs have recently become publicly available (Li et al. 2005; Dovčiak et al. 2005), and there now exists a fully-relativistic treatment of the effects of spectral hardening (Davis et al. 2005) . Applications of these models are discussed in $\S 8$. 
In parallel with these developments, MHD simulations have advanced our understanding of the nature of viscosity in accretion disks. The magnetorotational instability (MRI) has been shown to be a source of turbulent viscosity (Balbus \& Hawley 1991), a result that has been confirmed by several global GR MHD simulations (e.g., DeVilliers et al. 2003; McKinney \& Gammie 2004; Matsumoto et al. 2004). Investigators are now considering how MRI and MHD turbulence influences disk structure (e.g., Gammie 2004), the emerging thermal spectrum (e.g., Merloni 2003), and Comptonization effects (e.g., Socrates et al. 2004). MHD simulations of accretion disks are currently three-dimensional, global and based on the Kerr metric. They will soon include dissipative processes (i.e., radiation; see Johnson \& Gammie 2003), and it is hoped that they will then connect more directly with observation.

\subsection{Hard State}

The association of the hard state with the presence of a steady radio jet marked a substantial advance. Indirect signatures of this jet can now be recognized in the $\mathrm{X}$-ray data ( $\left.\S \$_{-}-5\right)$. However, the relationship between the disk and jet components and the origin of the X-ray properties of the hard state remain uncertain.

Difficulties in understanding the hard state are illustrated by results obtained for XTE J1118+480, a BHB with an extraordinarily small interstellar attenuation (e.g., only $30 \%$ at $0.3 \mathrm{keV}$ ) and a display of weak outbursts confined to the hard state. This source provides the best direct determination of the apparent temperature and radius of the inner disk in the hard state. Using simultaneous HST, EUVE, and Chandra observations (McClintock et al. 2001), the disk was found to be unusually large $\left(\sim 100 R_{\mathrm{g}}\right)$ and cool $(\sim 0.024 \mathrm{keV})$. Slightly higher temperatures $(\approx 0.035-0.052 \mathrm{keV})$ were inferred from observations with BeppoSAX (Frontera et al. 2003). Though it seems clear that the blackbody radiation is truncated at a large radius, the physical condition of material within this radius remains uncertain. Alternative scenarios include a thermal advection-dominated accretion flow (ADAF; Esin et al. 2001), a radiative transition to synchrotron emission in a relativistic flow that is entrained in a jet (Markoff et al. 2001), and a radiative transition to a Compton corona (Frontera et al. 2003), which must then be sufficiently optically thick to mask the $\sim 1 \mathrm{keV}$ thermal component normally seen from the disk. Such a corona might be a hot wind leaving the disk (Blandford \& Begelman 1999; 2004).

Recent investigations of other BHBs in the hard state suggest that both synchrotron and Compton components contribute to the broadband spectrum, with the Compton emission presumed to originate at the base of the jet (Kalemci et al. 2005; Markoff, Nowak, \& Wilms 2005). It is also possible that the jet is supplied by hot gas from a surrounding ADAF flow (Yuan, Cui, \& Narayan 2005).

Guidance in understanding the accretion flow in this inner region may eventually come from other types of investigations, such as the study of correlated optical/X-ray variability (Malzac et al. 2003). Also promising are spectral analyses that focus on features indicating densities higher than that expected for an optically thin flow, such as the ADAF mentioned above. One such feature is the broad Fe emission line (e.g., Miller et al. 2002b; 2002c). The Fe line profile can provide information on the Keplerian flow pattern and constrain the inner disk radius (see \$8.2.3). Another diagnostic spectral feature is an X-ray reflection component (Done \& Nayakshin 2001). In one study of the reflection compo- 
nent of Cyg X-1, the hard-state disk appeared to be truncated at a few tens of Schwarzschild radii (Done \& Zycki 1999).

\subsection{Steep Power Law State}

The physical origin of the SPL state remains one of the outstanding problems in high-energy astrophysics. It is crucial that we gain an understanding of this state, which is capable of generating HFQPOs, extremely high luminosity, and spectra that extend to $\gtrsim 1 \mathrm{MeV}$.

Most models for the SPL state invoke inverse Compton scattering as the operant radiation mechanism (see Zdziarski \& Gierliński 2004). The MeV photons suggest that the scattering occurs in a nonthermal corona, which may be a simple slab operating on seed photons from the underlying disk (e.g., Zdziarski et al. 2005). Efforts to define the origin of the Comptonizing electrons have led to models with more complicated geometry and with feedback mechanisms, such as flare regions that erupt from magnetic instabilities in the accretion disk (Poutanen \& Fabian 1999). There are alternative models of the SPL state. For example, bulk motion Comptonization has been proposed in the context of a converging sub-Keplerian flow within $50 R_{\mathrm{g}}$ of the BH (Titarchuk \& Shrader 2002; Turolla et al. 2002).

An analysis of extensive RXTE spectral observations of GRO J1655-40 and XTE J1550-564 shows that as the PL component becomes stronger and steeper, the disk luminosity and radius appear to decrease while the temperature remains high. These results can be interpreted as an observational confirmation of strong Comptonization of disk photons in the SPL state (Kubota \& Makishima 2004).

\section{$7.4 \quad Q P O$ Mechanisms}

In addition to spectral observations, it is also necessary to explain timing observations of LFQPOs $(0.1-30 \mathrm{~Hz})$, which are commonly seen in the SPL state. There are now a large number of proposed LFQPO mechanisms in the literature, and we mention only a few examples here. The models are driven by the need to account for both the observed range of frequencies and the fact that the oscillations are strongest at photon energies above $6 \mathrm{keV}$, i.e., where the PL component completely dominates over the disk component. The models include global disk oscillations (Titarchuk \& Osherovich 2000), radial oscillations of accretion structures such as shock fronts (Chakrabarti \& Manickam 2000), and oscillations in a transition layer between the disk and a hotter Comptonizing region (Nobili et al. 2000). Another alternative, known as the accretion-ejection instability model, invokes spiral waves in a magnetized disk (Tagger \& Pellat 1999) with a transfer of energy out to the radius where material corotates with the spiral wave. This model thereby combines magnetic instabilities with Keplerian motion to explain the observed QPO amplitudes and stability.

The behavior of the SPL state is complex and challenging. Nevertheless, we have much to work with, such as the exquisite quality of the data for this (usually) bright state, the regularities in behavior among various sources (Figures 4-9), and the remarkable couplings between the timing and spectral data (e.g., Figure 10). It appears that a successful model must allow for a highly dynamical interplay between thermal and nonthermal processes and involve mechanisms that can operate over a wide range of luminosity. Finally, we note that a physi- 
cal understanding of the SPL state is required as a foundation for building any complete model of the HFQPO mechanisms, a topic considered in further detail in $\S 8.2 .4$.

\section{Accreting Black Holes as Probes of Strong Gravity}

The continuing development of gravitational wave astronomy is central to the exploration of BHs. In particular, we can reasonably expect that LIGO and LISA will provide us with intimate knowledge concerning the behavior of space-time under the most extreme conditions. Nevertheless, gravitational wave detectors are unlikely to provide us with direct information on the formation of relativistic jets, on strong-field relativistic MHD accretion flows, or on the origin of high-frequency QPOs or broadened Fe lines. Accreting BHs - whether they be stellar-mass, supermassive or intermediate mass - promise to provide detailed information on all of these topics and more. In short, accreting BHs show us uniquely how a $\mathrm{BH}$ interacts with its environment. In this section, we first sketch a scenario for the potential impact of BHBs on physics, and we then discuss a current frontier topic, namely, the measurement of $\mathrm{BH}$ spin.

\subsection{Black Holes Binaries: The Journey from Astrophysics to Physics}

Astrophysics has a long history of impacting physics: e.g., Newton's and Einstein's theories of gravity, the ongoing research on dark matter and dark energy, the equation of state at supranuclear densities, and the solar neutrino puzzle. Likewise, astrophysical BHs have the potential to revolutionize classical BH physics; after all, the only real BHs we know, or are likely ever to know, are astrophysical BHs. But how can BHBs contribute to the study of BH physics? Very roughly, we envisage a five-stage evolutionary program that is presently well underway.

Stage I-Identify Dynamical BH Candidates: This effort is already quite advanced (see $\S 1$ and Table 1 ) and represents an important step because mass is the most fundamental property of a $\mathrm{BH}$. Nevertheless, the dynamical data do not probe any of the effects of strong gravity, and therefore we curtail the discussion of Stage I.

Stage II-Confirm that the Candidates are True Black Holes: Ideally, in order to show that a dynamical BH candidate (i.e., a massive compact object) is a genuine $\mathrm{BH}$, one would hope to demonstrate that the candidate has an event horizon - the defining characteristic of a BH. Strong evidence has been obtained for the reality of the event horizon from observations that compare BHBs with very similar neutron-star binaries. These latter systems show signatures of the hard surface of a neutron star that are absent for the BH systems. For example, $\mathrm{X}$-ray observations in quiescence show that the BH systems are about 100 times fainter than the nearly identical neutron-star binaries (Narayan et al. 1997; Garcia et al. 2001). The ADAF model ( $(7)$ provides a natural explanation for the faintness of the BHs, namely, the low radiative efficiency of the accretion flow allows a BH to "hide" most of its accretion energy behind its event horizon (e.g, Narayan et al. 2002). In quiescence, one also observes that BHs lack a soft thermal component of emission that is very prevalent in the spectra of neutron stars and can be ascribed to surface emission (McClintock, Narayan, \& Rybicki 2004). During outburst, the presence of a surface for an accreting neutron star 
likewise gives rise to distinctive phenomena that are absent in BHBs: $(a)$ type I thermonuclear bursts (Narayan \& Heyl 2002; Tournear et al. 2003; Remillard et al. 2006a), (b) high-frequency timing noise (Sunyaev \& Revnivtsev 2000), and (c) a distinctive spectral component from a boundary layer at the stellar surface (Done \& Gierliński 2003).

Of course, all approaches to this subject can provide only indirect evidence for the event horizon because it is quite impossible to detect directly any radiation from this immaterial surface of infinite redshift. Nevertheless, barring appeals to very exotic physics, the body of evidence just considered makes a strong case that dynamical $\mathrm{BH}$ candidates possess an event horizon.

Stage III-Measure the Spins of Black Holes: An astrophysical BH is described by two parameters, its mass $M$ and its dimensionless spin parameter $a_{*}$. Because the masses of $20 \mathrm{BHs}$ have already been measured or constrained (see Stage I and $\S 2$ ), the next obvious goal is to measure spin. Indeed, several methods to measure spin have been described in the literature, and various estimates of $a_{*}$ have been published, although few results thus far can be described as credible. We consider this stage to be a central and active frontier in $\mathrm{BH}$ research. Consequently we return to this subject below, where we discuss four approaches to measuring spin and some recent results for two BHBs.

Stage IV - Relate Black Hole Spin to the Penrose Process and Other Phenomena: A number of phenomena observed in astrophysical BHs have been argued to be associated with $\mathrm{BH}$ spin. The most notable examples are the explosive and relativistic radio jets associated with the hard-to-soft X-ray transition that occurs near the jet line (§4.3). Such ejections have been observed for at least eight BHBs and BHCs (Mirabel \& Rodriguez 1999; Fender \& Belloni 2004; McClintock \& Remillard 2006 and references therein). Also, large-scale relativistic X-ray jets have been reported for XTE J1550-564 (Hannikainen et al. 2001; Corbel et al. 2002) and H 1743-322 (Corbel et al. 2005). For many years, scientists have speculated that these jets are powered by $\mathrm{BH}$ spin via something like the Penrose (1969) process, which allows energy to be milked from a spinning BH. Detailed models generally invoke magnetic fields (Blandford \& Znajek 1977; Hawley \& Balbus 2002; Meier 2003; McKinney \& Gammie 2004). A number of beautiful ideas have been published along these lines, but there has been no way of testing or confirming them. Recently, however, some progress has been made on measuring $\mathrm{BH}$ spin, and it may soon be possible to attack the jet-spin/Penrose-process connection in earnest.

Stage V-Carry out Quantitative Tests of the Kerr Metric: One of the most remarkable predictions of $\mathrm{BH}$ physics is that the space-time surrounding a stationary rotating $\mathrm{BH}$ is described by the Kerr metric, which is completely specified by just two parameters, $M$ and $a_{*}$. Testing this prediction is the most important contribution astrophysics can make to BH physics. Obviously, in order to carry out such a test, one must first measure $M$ and $a_{*}$ with sufficient precision (Stage III). Once suitable measurements of $M$ and $a_{*}$ have been amassed for a number of BHs, we presume that astronomer's will be strongly motivated to devise ways of testing the metric, a topic which is beyond the scope of this work.

\subsection{Measuring Black Hole Spin: A Current Frontier}

We now elaborate on Stage III by discussing four avenues for measuring BH spin. These include (a) X-ray polarimetry, which appears very promising but 
thus far has not been incorporated into any contemporary X-ray mission; $(b)$ $\mathrm{X}$-ray continuum fitting, which is already producing useful results; $(c)$ the Fe K line profile, which has also yielded results, although the method is hampered by significant uncertainties; and $(d)$ high-frequency QPOs, which arguably offer the most reliable measurement of spin once a model is established. We now consider each of these in turn.

\subsubsection{Polarimetry}

As pointed out by Lightman \& Shapiro (1975) and Meszaros et al. (1988), polarimetric information (direction and degree) would increase the parameter space used to investigate compact objects from the current two (spectra and time variability) to four independent parameters that models need to satisfy. Such constraints are likely to be crucial in our attempts to model the hard state with its radio jet and the SPL state. However, because of the complexities of the accretion flows associated with these states $(\S 4, \S 5, \& \S 7)$ it appears unlikely that their study will soon provide quantitative probes of strong gravity. We therefore focus on disk emission in the thermal state.

The polarization features of $\mathrm{BH}$ disk radiation can be affected strongly by GR effects (Connors et al. 1980). The crucial requirement for a simple interpretation is that higher energy photons come from smaller disk radii, as they are predicted to do in conventional disk models $(\S 7)$. If this requirement is met, then as the photon energy increases from $1 \mathrm{keV}$ to $30 \mathrm{keV}$, the plane of linear polorization swings smoothly through an angle of about $40^{\circ}$ for a $9 M_{\odot}$ Schwarzschild $\mathrm{BH}$ and $70^{\circ}$ for an extreme Kerr BH (Connors et al. 1980). The effect is due to the strong gravitational bending of light rays. In the Newtonian approximation, on the other hand, the polarization angle does not vary with energy, except for the possibility of a sudden $90^{\circ}$ jump (Lightman \& Shapiro 1976). Thus, a gradual change of the plane of polarization with energy is a purely relativistic effect, and the magnitude of the change can give a direct measure of $a_{*}$.

A model is now available in XSPEC that allows one to compute the Stokes parameters of a polarized accretion disk spectrum (Dovčiak et al. 2004). While the theoretical picture is bright, and very sensitive instruments can be built (e.g., Kaaret et al. 1994; Costa et al. 2001), unfortunately, results to date are meager and there are no mission opportunities on the horizon. Important advances in this promising area could be made by a relatively modest mission given that BHBs in the thermal state are bright.

\subsubsection{Continuum Fitting}

Pioneering work in fitting the spectrum of the X-ray continuum to measure spin was carried out by Zhang et al. (1997a), and the method was advanced further by Gierliński et al. (2001). Very recently, two developments have allowed this method to be applied more widely and with some confidence, namely: (1) models of thin accretion disks are now publicly available in XSPEC ("KERRBB", Li et al. 2005; "KY", Dovčiak et al. 2004) that include all relativistic effects and allow one to fit for $a_{*}$; and (2) sophisticated disk atmosphere models now exist for computing the spectral hardening factor, $f_{\text {col }}=T_{\text {col }} / T_{\text {eff }}$ as a function of the Eddington-scaled luminosity of the disk (Davis et al. 2005).

This method of measuring $a_{*}$ depends on the properties of thin accretion disks 
described in $\S 7$ and is most convincing when it is applied to BHBs in the thermal state $(\S 4, \S 5, \& \S 7)$. Effectively, in this technique, one determines the radius $R_{\text {in }}$ of the inner edge of the accretion disk and assumes that this radius corresponds to $R_{\mathrm{ISCO}}$ (see $\S 1$ ). Because $R_{\mathrm{ISCO}} / R_{\mathrm{g}}$ is a monotonic function of $a_{*}$, a measurement of $R_{\text {in }}$ and $M$ directly gives $a_{*}$. Provided that $(a) i$ and $D$ are sufficiently well known, $(b)$ the X-ray flux and spectral temperature are measured from well calibrated X-ray data in the thermal state, and $(c)$ the disk radiates as a blackbody, it is clear that $R_{\text {in }}$ can be estimated. A major complication is that the disk emission is not a true blackbody but a modified blackbody with a spectral hardening factor $f_{\text {col }}$. Therefore, the observations only give the quantity $R_{\text {in }} / f_{\text {col }}^{2}$, and one needs an independent estimate of $f_{\text {col }}$ in order to estimate $a_{*}$ (Shimura \& Takahara 1995; Davis et al. 2005). A second caveat is that the orbital inclination may differ significantly from the inclination of the BH's spin axis (Maccarone 2002).

Using the new disk models mentioned above, Shafee et al. (2006) fitted ASCA and RXTE spectral data on the BHB GRO J1655-40 and found $a_{*} \sim 0.65-0.75$. For $4 \mathrm{U} 1543-47$, they found $a_{*} \sim 0.75-0.85$, although this result is based only on RXTE data. The authors consider it unlikely that either BH has a spin close to the theoretical maximum, $a_{*}=1$. On the other hand, in the case of $4 \mathrm{U} 1543-47$, the estimated spin appears too high to be explained by spin up due to accretion, which suggests that their measurements are sensitive to the BH's natal spin.

\subsubsection{Fe K line profile}

The first broad Fe K $\alpha$ line observed for either a BHB or an AGN was reported in the spectrum of Cyg X-1 based on EXOSAT data (Barr et al. 1985). Since then, the line has been widely studied in the spectra of both BHBs and AGN. The Fe $\mathrm{K}$ fluorescence line is thought to be generated through the irradiation of the cold (weakly-ionized) disk by a source of hard X-rays (likely an optically-thin, Comptonizing corona). Relativistic beaming and gravitational redshifts in the inner disk region can serve to create an asymmetric line profile (for a review, see Reynolds \& Nowak 2003).

The line has been modeled in the spectra of several BHBs. In some systems the inner disk radius deduced from the line profile is consistent with the $6 R_{\mathrm{g}}$ radius of the ISCO of a Schwarzschild BH, suggesting that rapid spin is not required (e.g., GRS 1915+105, Martocchia et al. 2002; V4641 Sgr, Miller et al. 2002a). On the other hand, fits for GX 339-4 indicate that the inner disk likely extends inward to $(2-3) R_{\mathrm{g}}$, implying $a_{*} \geq 0.8-0.9$ (Miller et al. 2004). XTE J1650-500 is the most extreme case with the inner edge located at $\approx 2 R_{\mathrm{g}}$, which suggests nearly maximal spin (Miller et al. 2002c; Miniutti et al. 2004). Large values of $a_{*}$ have also been reported for XTE J1655-40 and XTE J1550-564 (Miller et al. 2005). Sources of uncertainty in the method include the placement of the continuum, the model of the fluorescing source, and the ionization state of the disk (Reynolds \& Nowak 2003). Also, thus far the analyses have been done using the LAOR model in XSPEC, which fixes the spin parameter at $a_{*}=0.998$ (Laor 1991). A reanalysis of archival data using new XSPEC models that allow one to fit for $a_{*}$ may prove useful (KY, Dovčiak et al. 2004; KD, Beckwith \& Done 2004). 


\subsubsection{High Frequency Quasi-Periodic Oscillations}

Arguably, HFQPOs (see §6.2) are likely to offer the most reliable measurement of spin once the correct model is known. Typical frequencies of these fast QPOs, e.g., 150-450 Hz, correspond respectively to the frequency at the ISCO for Schwarzschild BHs with masses of $15-5 M_{\odot}$, which in turn closely matches the range of observed masses (Table 1). As noted in $\S 6.2$, these QPO frequencies (single or pairs) do not vary significantly despite sizable changes in the X-ray luminosity. This suggests that the frequencies are primarily dependent on the mass and spin of the BH. Those BHs that show HFQPOs and have well-constrained masses are the best prospects for contraining the value of the $\mathrm{BH}$ spin $\left(a_{*}\right)$.

The four sources that exhibit harmonic pairs of frequencies in a $3: 2$ ratio (see $§ 6.2$ ) suggest that HFQPOs arise from some type of resonance mechanism (Abramowicz \& Kluźniak 2001; Remillard et al. 2002a). Resonances were first discussed in terms of specific radii where particle orbits have oscillation frequencies in GR (see Merloni et al. 1999) that scale with a 3:1 or a 3:2 ratio. Current resonance concepts now consider accretion flows in a more realistic context. For example, the "parametric resonance" concept (Abramowicz et al. 2003; Kluzniiak et al. 2004; Török et al. 2005) describes oscillations rooted in fluid flow where there is a coupling between the radial and polar coordinate frequencies. As a second example, one recent MHD simulation provides evidence for resonant oscillations in the inner disk (Kato 2004); in this case, however, the coupling relation involves the azimuthal and radial coordinate frequencies. If radiating blobs do congregate at a resonance radius for some reason, then ray tracing calculations have shown that GR effects can cause measurable features in the X-ray power spectrum (Schnittman \& Bertschinger 2004).

Other models utilize variations in the geometry of the accretion flow. For example, in one model the resonance is tied to an asymmetric structure (e.g., a spiral wave) in the inner accretion disk (Kato 2005). In an alternative model, state changes are invoked that thicken the inner disk into a torus; the normal modes (with or without a resonance condition) can yield oscillations with a 3:2 frequency ratio (Rezzolla et al. 2003; Fragile 2005). All of this research is still in a developmental state, and these proposed explanations for HFQPOs are basically dynamical models that lack radiation mechanisms and fail to fully consider the spectral properties of HFQPOs described in $\S 6.2$.

Theoretical work aimed at explaining HFQPOs is motivated by the following empirical result that is based on a very small sample of three sources: XTE J1550564, GRO J1655-40 and GRS 1915+105. These sources are presently the only ones that both exhibit harmonic (3:2) HFQPOs and have measured $\mathrm{BH}$ masses. As shown in Figure 12, their frequencies appear to scale inversely with mass (McClintock \& Remillard 2006), which is the dependence expected for coordinate frequencies (see Merloni et al. 1999) or for diskoseismic modes in the inner accretion disk (Wagoner 1999; Kato 2001). If these HFQPOs are indeed GR oscillations, then Figure 12 further suggests that the three BHs have similar values of the spin parameter $a_{*}$. Obviously it is of great importance to attempt to confirm this result by obtaining the requisite frequency and mass measurements for additional sources. 


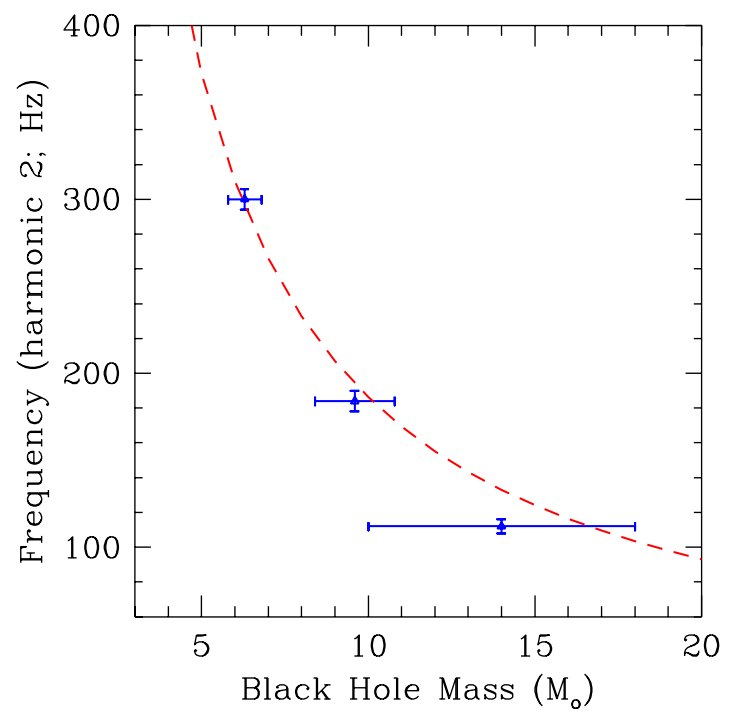

Figure 12: Relationship between high-frequency quasi-periodic oscillation (HFQPO) frequency and black hole mass for XTE J1550-564, GRO J1655-40, and GRS 1915+105. These three systems display a pair of HFQPOs with a 3:2 frequency ratio. The frequencies are plotted for the stronger QPO that represents $2 \times \nu_{0}$. The fundamental is generally not seen in the power spectra. The dashed line shows a relation, $\nu_{0}(\mathrm{~Hz})=931\left(M / M_{\odot}\right)^{-1}$, that fits these data.

\subsection{Critique of Methods for Measuring Spin}

In short, there are four avenues for measuring spin - polarimetry, continuum fitting, the Fe K line, and HFQPOs. Because spin is such a critical parameter, it is important to attempt to measure it by as many of these methods as possible, as this will provide arguably the best possible check on our results. The best current method, continuum fitting, has the drawback that its application requires accurate estimates of $\mathrm{BH}$ mass $(M)$, disk inclination $(i)$, and distance. In contrast, assuming we have a well-tested model, QPO observations require knowledge of only $M$ to provide a spin estimate. Broadened Fe $\mathrm{K}$ lines and polarimetry data do not even require $M$, although knowledge of $i$ is useful in order to avoid having to include that parameter in the fit. On the other hand, the Fe-line and HFQPO methods are not well-enough developed to be applied to real systems, and the required polarimetry data are not available, whereas the continuum method, despite its limitations, is already delivering results.

\section{Black-Hole Binaries Yesterday, Today and Tomorrow}

Three historic milestones in stellar $\mathrm{BH}$ research are separated by roughly one human generation, namely, Einstein's 1915 paper on GR, Oppenheimer and Snyder's 1939 paper on gravitational collapse, and the 1972 identification of BH Cyg $\mathrm{X}-1$ (see $\S 1$ ). Now, one more generation later, we have measured or constrained 
the masses of 20 BHBs and obtained spin estimates for two of them. As described herein, a rich X-ray data archive, as well as ongoing observations, are providing an intimate look at the behavior of transient BHs as they vary in X-ray luminosity by $5-8$ orders of magnitude. We have obtained strong circumstantial evidence for the existence of the event horizon, and observed harmonic pairs of HFQPOs and relativistically-broadened Fe lines emanating from near the ISCO. However, the RXTE detectors are unable to resolve the Fe K line, and the HFQPOs are all

near the limiting sensitivity of the mission. Further major advances will require a new timing mission with order-of-magnitude increases in detector area, telememetry capability, and spectral resolving power. It is important to press ahead with a new timing mission soon in order to complement effectively the vigorous programs underway in gravitational wave astronomy and in observational studies of supermassive and intermediate-mass BHs.

\section{Acknowledgements; Acronyms; Section Summaries}

\section{Acknowledgements}

We are grateful to Ramesh Narayan, Jerry Orosz, Rob Fender, and Alan Levine for contributing material for this review. We also thank Marek Abramowicz, Wlodek Kluźniak, Omer Blaes, Jeroen Homan, Phil Kaaret, and Jon Miller for helpful disussions while this paper was in preparation. This work was funded in part by the NASA contract to MIT for support of RXTE and also by NASA Grant NNG-05GB31G to JEM.

\section{Acronyms}

BH: Black hole

BHB: Black hole binary

BHC: Black hole candidate

HID: Hardness intensity diagram

GR: General relativity

HR: Hardness ratio

ISCO: Innermost stable circular orbit

PDS: Power density spectrum

QPO: Quasi-periodic oscillation

PL: Power-law

\section{Summary Points}

1. The topics of black-hole binaries and candidates are introduced, along with the perspective of general relativity on black hole mass and spin.

2. The presence of a black hole is deduced from dynamical measurements of its binary companion. Twenty such systems are identified.

3. Data analysis techniques are summarized for the X-ray observations that characterize black holes.

4. Accreting black holes exhibit X-ray states that are seen as distinct and very 
different combinations of X-ray energy spectra and power density spectra.

5. The temporal evolution of X-ray states and the manner in which states are related to primary spectral and timing properties are illustrated for six selected sources.

6. Quasi-periodic oscillations occur in some states. They span a wide range in frequency, and they impose requirements on physical models.

7. Physical models are briefly reviewed for X-ray states and quasi-periodic oscillations.

8. We discuss the present and future for efforts to utilize black-hole binaries as a test bed for applications of general relativity.

Literature Cited

Abramowicz MA, Karas V, Kluźniak W, Lee WH, Rebusco P 2003. PASJ 55:467-71

Abramowicz MA, Kluźniak W 2001. Astron. Astrophys. 374:L19-20

Agol E, Krolik JH 2000. Ap. J. 528:161-70

Balbus SA, Hawley JF 1991. Ap. J. 376:214-22

Barr P, White NE, Page CG 1985. MNRAS 216:65P-70P

Beckwith K, Done C 2004. MNRAS 352: 353-62

Belloni T 2004. NuPhS 337-45

Belloni T, Homan J, Casella P, van der Klis M, Nespoli E, et al. 2005. Astron. Astrophys. 440:207-22

Blandford RD, Begelman C. 1999. MNRAS 303:L1-5

Blandford RD, Begelman C. 2004. MNRAS 349:68-86

Blandford RD, Znajek RL 1977. MNRAS 179:433-56

Bolton, CT 1972. Nature 235:271-73

Brown GE, Bethe HA 1994. Ap. J. 423:659-64

Brown GE, Lee C-H, Wijers RAM, Lee HK, Israelian G, Bethe HA 2000. New Ast 5:191-210

Casares J, Zurita C, Shahbaz T, et al. 2004. Ap. J. 613:L133-36

Casella P, Belloni T, Homan J, Stella L 2004. Astron. Astrophys. 426:587-600

Casella P, Belloni T, Stella L 2005. Ap. J. 629:403-7

Chakrabarti SK, Manickam SG 2000. Ap. J. 531:L41-44

Charles PA, Coe MJ 2006. In Compact Stellar X-ray Sources, ed. WHG Lewin, M van der Klis, pp. 215-66. Cambridge: Cambridge University Press. (astro-ph/0308020).

Chen W, Shrader CR, Livio M 1997. Ap. J. 491:312-38

Coe MJ, Engel AR, Quenby JJ 1976. Nature 259:544-45

Connors PA, Piran RF, Stark T 1980. Ap. J. 235:224-44

Corbel S, Fender RP, Tzioumis AK, Nowak M, McIntyre V, et al. 2000. Astron. Astrophys. 359:251-68

Corbel S, Fender RP, Tzioumis AK, et al. 2002. Science 298:196-99

Corbel S, Kaaret P, Fender RP, Tzioumis AK, Tomsick JA, Orosz JA. 2005. Ap. J. 632:504-13

Corbel S, Tomsick JA, Kaaret P 2006. Ap. J. 636:971-78

Costa E, Soffitta P, Bellazzini R, Brez A, Lumb N, et al. 2001 Nature 411: 662-65

Cowley AP, Crampton D, Hutchings JB, Remillard R, Penfold JE 1983. Ap. J. 272:118-22

Cui W, Shrader CR, Haswell CA, Hynes RI 2000a. Ap. J. 535:L123-27

Cui W, Zhang SN, Chen W 2000b. Ap. J. 531:L45-48

Davis SW, Blaes OM, Hubeny I, Turner NJ 2005. Ap. J. 621:372-98

DeVilliers J-P, Hawley JF, Krolik JH 2003. Ap. J. 599:1238-53

Dhawan V, Mirabel IF, Rodriguez LF 2000. Ap. J. 543:373-85

Done C, Gierliński M 2003. MNRAS 342:1041-55

Done C, Nayakshin S 2001. MNRAS 328:616-22

Done C, Zycki PT 1999. MNRAS 305:457-68

Dovčiak M, Karas V, Yaqoob T 2004. Ap. J. Supp. 153:205-21

Dubus G, Hameury J-M, Lasota J-P 2001. Astron. Astrophys. 373:251-71

Elvis M, Page CG, Pounds KA, Ricketts MJ, Turner MJL 1975. Nature 257:656-57

Esin AA, McClintock JE, Drake JJ, Garcia MR, Haswell CA, et al. 2001. Ap. J. 555:483-88 
Fender RP 2006. In Compact Stellar X-ray Sources, ed. WHG Lewin, M van der Klis, pp. 381420. Cambridge: Cambridge University Press. (astro-ph/0303339).

Fender RP, Belloni T 2004. Annu. Rev. Astron. Astrophys. 42: 317-64

Fender RP, Belloni T, Gallo E 2004. MNRAS 355:1105-18

Fender R, Corbel S, Tzioumis T, McIntyre V, Campbell-Wilson D, et al. 1999. Ap. J. 519:L16568

Fragile CP. 2005. In Proceedings of 22nd Texas Symposium on Relativistic Astrophysics at Stanford", ed. P Chen, E Bloom, G Madejski, V Patrosian, econf/C041213. (astro-ph/0503305)

Frontera F, Amati L, Zdziarski AA, Belloni T, Del Sordo S, et al. 2003. Ap. J. 592: 1110-18

Fryer C, Kalogera V 2001. Ap. J. 554:548-60

Gallo E, Fender RP, Pooley GG 2003. MNRAS 344:60-72

Gammie CF 2004 Ap. J. 614:309-13

Garcia MR, McClintock JE, Narayan R, Callanan P, Barret D 2001. Ap. J. 553:L47-50

Gierliński M, Maciolek-Niedzwiecki A, Ebisawa K 2001. MNRAS 325:1253-65

Gliozzi M, Papadakis IE, Räth C 2006. Astron. Astrophys. in press. (astro-ph/0512026)

Grove JE, Johnson WN, Kroeger RA, et al. 1998. Ap. J. 500:899-908

Hannikainen D, Campbell-Wilson D, Hunstead R, et al. 2001. Ap. Space Sci. 276:45-48

Hawley, JF, Balbus SA. 2002. Ap. J. 573:738-48

Homan J, Klein-Wolt M, Rossi S, Miller JM, Wijnands R, et al. 2003. Ap. J. 586:1262-67

Homan J, Miller JM, Wijnands R, Lewin WHG. 2005a. BAAS 207:1331-31

Homan J, Miller JM, Wijnands R, van der Klis M, Belloni T, et al. 2005b. Ap. J. 623:383-91

Homan J, Wijnands R, van der Klis M, Belloni T, van Paradijs J, et al. 2001. Ap. J. Supp. 132:377-402

Hynes RI, Steeghs D, Casares J, Charles PA, O'Brien K 2003. Ap. J. 583:L95-98

Israelian G, Rebolo R, Basri G 1999. Nature 401:142-44

Johnson BM, Gammie CF 2003. Ap. J. 597:131-41

Kaaret PE, Schwartz J, Soffitta P, Dwyer J, Shaw PS, et al. 1994 SPIE 2010:22-27

Kalemci E, Tomsick JA, Buxton MM, Rothschild RE, Pottschmidt K, et al. 2005. Ap. J. 622:50819

Kalogera V, Baym G 1996. Ap. J. 470:L61-64

Kato S. 2001. PASJ 53:1-24

Kato S. 2005. PASJ 57:L17-20

Kato S, Fukue J, Mineshige S 1998. Black-Hole Accretion Disks, Kyoto: Kyoto U Press. 594 pp.

Kato Y 2004. PASJ 56:931-37

Klein-Wolt M, Homan J, van der Klis M 2004. NuPhS 132..381K132: 381-86

Kluźniak W, Abramowicz MA, Lee W 2004. AIPC 714:379-82

Kong AKH, Charles PA, Kuulkers E, Kitamoto S 2002. MNRAS 329:588-96

Koyama K, Ikegami T, Inoue H, et al. 1984. PASJ 36:659-66

Kubota A, Ebisawa K, Makishima K, Nakazawa, K 2005. Ap. J. 631:1062-71

Kubota A, Makishima K, 2004. Ap. J. 601:428-38

Laor A 1991. Ap. J. 376:90-94

Lasota J-P 2001. New Astron Rev 45:449-508

Leahy DA, Darbro W, Elsner RF, Weisskopf MC, Sutherland PG, et al. 1983. Ap. J. 266:160-70

Lee JC, Reynolds CS, Remillard RA, Schulz NS, Blackman EG, Fabian AC 2002. Ap. J. 567:1102-11

Levine AM, Bradt H, Cui W, Jernigan JG, Morgan, EH, et al. 1996. Ap. J. 469:33-36

Li L-X, Zimmerman ER, Narayan R, McClintock JE 2005. Ap. J. Supp. 157:335-70

Lightman AP, Shapiro SL 1975. Ap. J. 198: L73-75

Lightman AP, Shapiro SL 1976. Ap. J. 203:701-3

Maccarone TJ, Coppi PS 2003. MNRAS 338:189-96

Maccarone TJ 2002. MNRAS 336: 1371-76

Makishima K, Maejima ,Y Mitsuday K, Bradt HV, Remillard, RA, et al. 1986. Ap. J. 308:635-43

Malzac J, Belloni T, Spruit HC, Kanbach G 2003. Astron. Astrophys. 407: 335-45

Markoff S, Falcke H, Fender R 2001. Astron. Astrophys. 372:L25-28

Markoff S, Nowak MA, Wilms J. 2005. Ap. J. 635:1203-16

Martocchia A, Matt G, Karas V, Belloni T, Feroci M 2002. Astron. Astrophys. 387: 215-21

Matsumoto R, Machida M, Nakamura K. 2004. PThPS 155:121-31

McClintock JE, Haswell CA, Garcia MR, Drake JJ, Hynes RI, et al. 2001. Ap. J. 555:477-82

McClintock JE, Horne K, Remillard RA 1995. Ap. J. 442:358-65 
McClintock JE, Narayan R., Rybicki GB 2004. Ap. J. 615:402-15

McClintock JE, Remillard RA 1986. Ap. J. 308:110-22

McClintock JE, Remillard RA 2006. In Compact Stellar X-ray Sources, ed. WHG Lewin, M van der Klis, pp. 157-214. Cambridge: Cambridge University Press. (astro-ph/0306213)

McKinney JC, Gammie CF 2004. Ap. J. 611:977-95

Meier D 2003 NewAR 47:667-72

Merloni A 2003. MNRAS 341:1051-56

Merloni A, Vietri M, Stella L, Bini D 1999. MNRAS 304:155-59

Meszaros P, Novick R, Szentgyorgyi A, Chanan GA, Weisskopf MC 1988. Ap. J. 324: 1056-67

Miller JM, Fabian AC, in't Zand JJM, Reynolds CS, Wijnands R. et al. 2002a. Ap. J. 577:L15-18

Miller JM, Fabian AC, Nowak MA, Lewin W 2005. Procs., Tenth Marcel Grossmann Meeting on

General Relativity, ed. M Novello, S Perez-Bergliaffa, R Ruffini, in press. Singapore: World

Scientific. (astro-ph/0402101)

Miller JM, Fabian AC, Reynolds CS, Nowak MA, Homan J, et al. 2004. Ap. J. 606: L131-34

Miller JM, Fabian AC, Wijnands R, Remillard RA, Wojdowski P, et al. 2002b. Ap. J. 578:348-56

Miller JM, Fabian AC, Wijnands R, Reynolds CS, Ehle M, et al. 2002c. Ap. J. 570:L69-73

Miller JM, Wijnands R, Homan J, Belloni T, Pooley GG, et al. 2001. Ap. J. 563:928-33

Miniutti G, Fabian AC, Miller JM 2004. MNRAS 351: 466-72

Mirabel IF, Rodriguez LF 1999. Annu. Rev. Astron. Astrophys. 37:409-43

Mitsuda K, Inoue H, Koyama K, Makishima K, Matsuoka M, et al. 1984. PASJ 36:741-59

Miyamoto S, Iga S, Kitamoto S, Kamado Y 1993. Ap. J. 403:L39-42

Miyamoto S, Kitamoto S 1991. Ap. J. 374:741-43

Morgan EH, Remillard RA, Greiner J 1997. Ap. J. 482:993-10

Muno MP, Morgan EH, Remillard RA 1999. Ap. J. 527:321-40

Muno MP, Morgan EH, Remillard RA, et al. 2001. Ap. J. 556:515-32

Muno MP, Remillard RA, Chakrabarty D 2002. Ap. J. 568:L35-39

Narayan R, Garcia MR, McClintock JE 1997. Ap. J. 478:L79-82

Narayan R, Garcia MR, McClintock JE 2002. In Proc Ninth Marcel Grossmann Meeting, ed.

VG Gurzadyan, et al. pp. 405-5. Singapore: World Scientific

Narayan R, Heyl JS 2002. Ap. J. 574:L139-42

Nobili L, Turolla R, Zampieri L, Belloni T 2000. Ap. J. 538:L137-40

Nowak MA 2000. MNRAS 318:361-67

Oppenheimer JR, Snyder H 1939. Phys Rev 56:455-59

Orosz JA 2003. In A Massive Star Odyssey: From Main Sequence to Supernova, ed. KA van der Hucht, A Herraro, C Esteban, pp. 365-71. San Francisco: Astronomical Society of the Pacific. (astro-ph/0209041)

Orosz JA, Kuulkers E, van der Klis M, McClintock JE, Garcia MR, et al. 2001. Ap. J. 555:489503

Orosz JA, McClintock JE, Remillard RA, Corbel S 2004. Ap. J. 616: 376-82

Park SQ, Miller JM, McClintock JE, Remillard RA, Orosz JA, et al. 2004. Ap. J. 610:378-89

Penrose R 1969. Riv Nuovo Cim 1:252-76

Podsiadlowski P, Rappaport S., Han A 2003. MNRAS 341:385-404

Poutanen J, Fabian AC, 1999. MNRAS 306:L31-37

Pringle JE 1981. Annu. Rev. Astron. Astrophys. 19:137-62

Psaltis D. 2006. In Compact Stellar X-ray Sources, ed. WHG Lewin, M van der Klis, pp. 1-38.

Cambridge: Cambridge University Press. (astro-ph/0410536).

Remillard RA 2004. AIPC 714:13-20

Remillard RA, Lin D, Cooper R, Narayan R. 2006a. Ap. J. in press (vol. 646; astro-ph/0509758)

Remillard RA, McClintock JE, Orosz JA, Levine AM 2006b. Ap. J. 637:1002-9

Remillard RA, Morgan EH, McClintock JE, Bailyn CD, Orosz JA 1999. Ap. J. 522:397-412

Remillard RA, Muno MP, McClintock JE, Orosz JA 2002a. Ap. J. 580:1030-42

Remillard RA, Sobczak GJ, Muno MP, McClintock JE 2002b. Ap. J. 564:962-73

Revnivtsev M, Trudolyubov SP, Borozdin KN 2000. MNRAS 312:151-58

Reynolds CS, Nowak MA 2003. PhysRept 377:389-466

Rezzolla L, Yoshida S'i, Maccarone TJ, Zanotti O 2003. MNRAS 344:L37-41

Rodriguez J, Durouchoux P, Mirabel IF, Ueda Y, Tagger M, Yamaoka K 2002. Astron. Astrophys. 386:271-79

Rossi S, Homan J, Miller JM, Belloni T 2004. NuPhS 132:416-19

Scargle JD 1981. Ap. J. Supp. 45:1-71 
Shafee R, McClintock JE, Narayan R, Davis SW, Li L-X, Remillard RA 2006. Ap. J. 636:L11316

Shakura NI, Sunyaev RA 1973. Astron. Astrophys. 24:337-66

Shapiro SL, Teukolsky SA 1983. Black Holes White Dwarfs and Neutron Stars: The Physics of Compact Objects. New York: Wiley. 645 pp.

Shimura T, Takahara F 1995. Ap. J. 445:780-88

Smak J 1971. Acta Astron 21:15-21

Sobczak GJ, McClintock JE, Remillard RA, Bailyn CD, Orosz JA 1999. Ap. J. 520:776-87

Sobczak GJ, McClintock JE, Remillard RA, Cui W, Levine AM, et al. 2000a. Ap. J. 531:537-45

Sobczak GJ, McClintock JE, Remillard RA, Cui W, Levine AM, et al. 2000b. Ap. J. 544:9931015

Socrates A, Davis SE, Blaes O 2004 Ap. J. 601:405-13

Stella L, Vietri M, Morsink SM 1999. Ap. J. 524:L63-66

Stirling AM, Spencer RE, de la Force CJ, et al. 2001. MNRAS 327:1273-78

Strohmayer TE 2001a. Ap. J. 552:L49-53

Strohmayer TE 2001b. Ap. J. 554:L169-72

Sunyaev R, Revnivtsev M 2000. Astron. Astrophys. 358:617-23

Swank J 1998. NuPhS 69:12-19

Tagger M, Pellat R 1999. Astron. Astrophys. 349:1003-16

Tanaka Y, Lewin WHG 1995. In X-ray Binaries, ed. WHG Lewin, J van Paradijs, EPJ van den Heuvel, pp. 126-74. Cambridge: Cambridge U Press

Tanaka Y, Shibazaki N 1996. Annu. Rev. Astron. Astrophys. 34:607-44

Tananbaum H, Gursky H, Kellogg E, et al. 1972. Ap. J. 177:L5-10

Timmes FX, Woosley SE, Weaver TA 1996. Ap. J. 457:834-43

Titarchuk L, Osherovich V 2000. Ap. J. 542:L111-14

Titarchuk L, Shrader C 2002. Ap. J. 567:1057-66

Tomsick JA, Kaaret P 2001. Ap. J. 548:401-9

Tomsick JA, Kaaret P, Kroeger RA, Remillard RA 1999. Ap. J. 512:892-900

Török G, Abramowicz MA, Kluźniak W, Stuchlík Z 2005. Astron. Astrophys. 436:1-8

Tournear D, Raffauf E, Bloom ED, Focke W, Giebels B, et al. 2003. Ap. J. 595: 1058-65

Turolla R, Zane S, Titarchuk L 2002. Ap. J. 576:349-56

van der Klis M 2006. In Compact Stellar X-ray Sources, ed. WHG Lewin, M van der Klis, pp. 39-112. Cambridge: Cambridge University Press. (astro-ph/0410551).

van Paradijs, J McClintock JE 1995. In X-ray Binaries, ed. WHG Lewin, J van Paradijs, EPJ van den Heuvel, pp. 58-125. Cambridge: Cambridge U Press.

Vaughan BA, Nowak MA 1997. Ap. J. 474:L43-46

Vignarca F, Migliari S, Belloni T, Psaltis D, van der Klis M 2003. Astron. Astrophys. 397:729-38

Wagoner RV 1999. Phys. Rept. 311:259-69

Webster BL, Murdin P 1972. Nature 235:37-38

White NE, Nagase F, Parmar AN 1995. In X-ray Binaries, ed. WHG Lewin, J van Paradijs, EPJ van den Heuvel, pp. 1-57. Cambridge: Cambridge U Press

Wijnands R, Homan J, van der Klis M 1999. Ap. J. 526:L33-36

Woosley SE, Heger A, Weaver TA 2002. RvMP 74:1015-71

Yuan F, Cui W, Narayan R. 2005. Ap. J. 620:905-14

Zdziarski AA, Gierliński M 2004. PThPS 155:99-119

Zdziarski AA, Gierliński M, Rao AR, Vadawale SV, Mikolajewska J 2005. MNRAS 360:825-38

Zhang SN, Cui W, Chen W 1997a. Ap. J. 482:L155-58

Zhang SN, Cui W, Harmon BA, et al. 1997b. Ap. J. 477:L95-98

Zimmerman ER, Narayan R, McClintock JE, Miller JM 2005. Ap. J. 618: 832-44 